\title{
Corela
}

Cognition, représentation, langage

10-1 | 2012

Vol. $10, \mathrm{n}^{\circ} 1$

\section{Une méthode prosodique et linguistique pour générer une parole subjective en synthèse}

\section{Geneviève Caelen-Haumont}

\section{OpenEdition}

\section{Journals}

Édition électronique

URL : https://journals.openedition.org/corela/2686

DOI : $10.4000 /$ corela.2686

ISSN : 1638-573X

Éditeur

Cercle linguistique du Centre et de I'Ouest - CerLICO

\section{Référence électronique}

Geneviève Caelen-Haumont, « Une méthode prosodique et linguistique pour générer une parole subjective en synthèse », Corela [En ligne], 10-1 | 2012, mis en ligne le 12 juin 2012, consulté le 18 juin 2021. URL : http://journals.openedition.org/corela/2686 ; DOI : https://doi.org/10.4000/corela.2686

Ce document a été généré automatiquement le 18 juin 2021.

\section{cc) (i) (5)}

Corela - cognition, représentation, langage est mis à disposition selon les termes de la licence Creative Commons Attribution - Pas d'Utilisation Commerciale - Partage dans les Mêmes Conditions 4.0 International. 


\title{
Une méthode prosodique et linguistique pour générer une parole subjective en synthèse
}

\author{
Geneviève Caelen-Haumont
}

\section{Introduction : une affaire de subjectivité}

1 Les articles en linguistique concernant la prosodie sont relativement peu nombreux, et parmi eux, ceux qui tentent de franchir le fossé entre les analyses linguistiques et les analyses automatiques le sont encore moins. Rares sont donc de telles études, or les connaissances accumulées par les linguistes doivent nécessairement être appliquées aux recherches automatiques. C'est le but de cette communication qui issue d'une connaissance experte dans le domaine de la prosodie subjective accumulée depuis plusieurs décades, trouve ici son terme et son aboutissement. Naturellement cette communication qui explore le phénomène des mots subjectivement proéminents dans le discours s'adresse aussi aux linguistes et étudiants avancés.

2 Les liens entre analyse linguistique et synthèse de la parole se trouvent bien soulignés par Suciu et al. (2007): «le discours oral n'est pas un être composé de deux êtres antérieurs et autonomes (le texte et l'expressivité orale) mais un être à la fois premier et complet que l'on ne saurait décomposer qu'a posteriori et logiquement. La synthèse vocale, à l'assaut de services concrets mais contraints dans le calculable, suit obligatoirement ce même chemin logique et inverse pour penser ses réalisations ", et « en cherchant à mettre sur pied des briques technologiques incorporant de l'expressivité, la synthèse vocale ne peut longtemps ignorer ces enjeux, somme toute sémiotiques ». Ce programme défini dans le contexte des recherches à Orange Labs, constitue précisément notre propre contexte de recherche.

3 En discours spontané oral deux phénomènes se partagent le champ de la subjectivité : celui des émotions (et attitudes) et celui des proéminences prosodiques (ou mélismes). Ces phénomènes typiquement humains sont aussi importants l'un que l'autre dans 
l'expression de l'affect (Mac Dang, 2012). Les modèles de synthèse doivent donc s'en approcher au maximum pour améliorer leur expressivité subjective.

4 Le terme de mélisme que nous avons appliqué depuis 2000 aux mots possédant des modulations mélodiques particulières à caractérisation subjective (Caelen-Haumont et Bel, 2000) est emprunté à l'expression lyrique traditionnelle. Selon Wikipedia «le mélisme (du grec [...] melos, "air, mélodie, chant") est une technique consistant à charger de nombreuses notes une syllabe d'un texte, lorsque celui-ci est chanté. Cette musique est opposée à la syllabique, dans laquelle chaque syllabe du texte est fondue dans une seule note. Dans les mélodies traditionnelles, le mélisme naît de l'augmentation par broderie d'une mélodie initiale simple ». Cette définition s'applique bien aussi à certains mots rendus perceptuellement proéminents dans les énoncés de parole grâce à des modulations mélodiques spécifiques. Celles-ci réalisées spontanément par le locuteur sont l'objet de cette communication.

5 En analyse prosodique, il est du reste difficile de différencier les paramètres prosodiques qui relèvent du champ des émotions et de celui des mélismes. Prenons par exemple une émotion qui se rapproche le plus de l'expression du mélisme, à savoir la joie. Tous deux étant également caractérisés par une valence positive, c'est-à-dire manifestant un degré assez élevé d'excitation, les paramètres qui les caractérisent sont difficilement opposables: les valeurs de F0 aiguës, les contrastes de F0 importants, l'intensité élevée, les ruptures appartiennent à l'expression de l'un et de l'autre... Une étude récente (Caelen-Haumont, 2009) comparant les mélismes aux mots issus prononcés sous l'emprise de la joie et de l'hilarité, a conclu que les oppositions se trouvaient en fait à d'autres niveaux.

Ainsi les mélismes :

- sont fréquents en parole spontanée (en moyenne dans le corpus étudié, toutes les 6.9 syllabes, cf ci-dessous paragraphe 5.2.),

- ne concernent qu'un seul mot, au plus deux mots par contact généralement avec un mot grammatical, qui du fait de l'inertie des organes, hérite à sa frontière de gauche ou de droite selon sa position, de valeurs aiguës,

- n'ont de ce fait ni l'étoffe ni le statut nécessaires pour permettre d'exprimer vs. d'identifier un type d'émotion particulier,

- sont produits sous l'effet d'une conviction ou d'une croyance.

Inversement les segments de parole produits sous l'effet d'une émotion sont :

- incomparablement plus rares en langage standard,

- affectent des unités de discours beaucoup plus importantes (au minimum 4 ou 5 mots, mais parfois bien plus), et jamais un seul mot,

- correspondent à des changements d'états physiologiques, biologiques et cognitifs en réaction le plus souvent à un stimulus externe.

6 Ces deux phénomènes semblent donc distribués de manière complémentaire dans le discours spontané, avec des fréquences très différentes. Si en effet il est précisément de bon ton en société de maîtriser ou de masquer ses émotions lorsque l'on s'exprime, inversement les marques prosodiques d'un discours approprié par le locuteur où il se rend donc subjectivement présent, mettant en valeur les mots ressentis comme les plus importants, facilitent certainement la compréhension, l'échange discursif, et ne sont donc pas l'objet d'un préjugé social. En effet il faut beaucoup de confiance vis-à-vis d'un auditoire, ou être sous le joug d'une passion ou d'un intérêt très puissant pour donner libre cours à ses émotions (non jouées) devant un tiers ou un public. De la sorte ces 
conditions psychosociales éloignent souvent l'expression émotionnelle des applications d'aide à la personne, telles que celles du dialogue Homme-machine, et en particulier de la synthèse, même si les émotions en voix de synthèse sont requises dans le domaine de l'industrie des jeux.

Plus précisément les mélismes pointant les mots essentiels sur les plans sémantique, pragmatique et subjectif (Caelen-Haumont, 2009a en particulier), sont déterminants pour sa compréhension, la qualité du discours, son naturel, sa convivialité, et tout en prenant en charge discrètement l'attention d'autrui, ne sont pas trop intrusifs. A l'opposé, la manipulation émotionnelle est sans doute un des enjeux de cet autre domaine des nouvelles technologies des jeux et jouets, des robots et dessins animés. Dans ce domaine des technologies vocales et des jeux, en pleine expansion sinon explosion, de plus en plus de travaux sont ainsi investis à l'échelon international depuis une quinzaine d'années environ (Audibert, 2008 ; Le Beux et al., 2007).

8 L'étude de ce phénomène essentiel aux discours et aux dialogues spontanés qu'est le mélisme, toujours à l'œuvre dans un « discours approprié », semble donc à nos yeux un élément déterminant de l'expressivité subjective de la voix synthétique dans le plus grand nombre de ses applications. Par chance l'insertion des marqueurs prosodiques issus des mélismes est une procédure beaucoup plus facile (car plus limitée et bien circonscrite), que celle des émotions, domaine fort complexe. C'est pourquoi le phénomène des mélismes pourra être analysé dans ces pages sous toutes ses facettes en vue de la réalisation de cet objectif.

Soulignons que le but de cette étude n'est pas d'exposer des résultats en synthèse, mais en vue de ceux-ci, de proposer dans le domaine linguistique de la prosodie, une méthode nouvelle d'introduction de subjectivité en génération de parole, ou encore dans le cadre d'un discours naturel spontané jugé trop froid et inexpressif, de le transformer en un discours subjectivement investi (il s'agit alors selon le terme en usage de "resynthèse »). Précisément ce sont des exemples en resynthèse que nous avons générés en fonction des règles décrites dans ces pages, que nous soumettons à la perception auditive et visuelle. Ces exemples sont expliqués et commentés dans la partie Annexe de cette communication.

10 Comme indiqué ci-dessus (Suciu et al., 2007), le traitement de la parole subjective en synthèse s'élabore à plusieurs niveaux : à la fois à partir du texte, et bien entendu du traitement de la prosodie. A partir du texte, il faut tout d'abord isoler les mots qui seront le support de cette subjectivité, devenant par un traitement prosodique adéquat, des mélismes. Pour ce faire, il est capital de faire converger les objectifs du discours et le traitement prosodique. Les objectifs du discours sont très précocement traités par les structures cognitives, bien en amont des nombreux processus qui visent à la production de la parole, mais par nécessité, sont aussi traités conjointement au traitement prosodique, car les deux ont des racines subjectives.

11 Or ces objectifs du discours peuvent être révélés de manière explicite par les buts avoués par le locuteur ou exprimés par l'interlocuteur, ou s'ils sont implicites, peuvent être mis à jour par exemple grâce à une analyse des isotopies (Rastier, 1985 ; CaelenHaumont, 1991, 2009a). Nous reviendrons sur ce point au paragraphe 5.1..

12 Concernant le traitement de la prosodie subjective par les mélismes, objet de ces pages, nous essaierons d'en aborder tous ses éléments essentiels, vus de manière externe par rapport à la localisation des mélismes, leurs critères généraux, et de manière interne en 
plusieurs volets. Nous aborderons aussi les points de vue mélodique et temporel afin d'offrir les critères fondamentaux permettant de reproduire des mélismes dans la voix de synthèse de manière conforme à la voix naturelle. Comme annoncé ci-dessus, c'est donc un panorama complet que tente cette étude ${ }^{1}$. Cependant les aspects sémantiques et pragmatiques que nous avons amplement développés depuis l'origine de nos travaux (Caelen-Haumont 1978-1981；1991；2000；2002；2004; 2009a) ne seront pas développés dans ces pages car l'objectif de cette communication n'est pas orienté vers l'étude sémantique et pragmatique des mélismes, mais vers une méthode d'insertion des mélismes dans une parole de synthèse ${ }^{2}$.

Dans une première partie de l'étude présente (paragraphe 3 ci-dessous), nous décrivons les caractéristiques du corpus, et la banque de données qui a été construite. Cette banque de données fournira à la génération de parole le dictionnaire des patrons mélodiques et gabarits temporels. La deuxième partie (paragraphe 4 et suivants) centrée sur la prosodie, décrit le processus du mélisme de manière externe et interne. On y abordera les volets mélodique et temporel. La troisième partie (paragraphe 5) se focalise comme déjà précisé sur une description linguistique minimale (plans contextuel, morphosyntaxique et fonctions grammaticales), c'est-à-dire orientée seulement vers une description utile à la génération des mélismes. Enfin en annexe cette méthode de génération est expliquée et illustrée en détail.

\section{Brefs repères en synthèse de la parole}

Avant de commencer l'analyse, il peut être utile de proposer quelques repères dans le domaine de la synthèse de la parole en direction de la communauté des linguistes.

Au fil des années, et surtout depuis les années 1970, les systèmes de synthèse vocale se sont multipliés dans le contexte international, et se sont métamorphosés. Les principaux systèmes sont Infovox pour le suédois développé par Telia Promotor Infovox (Ljungqvist 1994), Acapela Group (Boula de Mareüil et al., 2001), qui depuis 2004 regroupe trois sociétés majeures européennes en technologie vocale: Babel Technologies (Belgique), Infovox (Suède) et Elan Speech (France), avec deux applications principales «Acapela telecom » conçu pour la synthèse de la parole multilingue dans le contexte des applications téléphoniques et des serveurs, et «Infovox desktop » développé pour synthétiser des textes sous Windows. AT\&T Natural Voices est un système multilingue pour l'anglais, le français, l'allemand et l'espagnol, sous également deux versions, pour ordinateur personnel et pour applications téléphoniques. Le groupe américain Nuance (www.nuance.com), est un des tout premiers leaders mondiaux dans le domaine des technologies vocales (et images). Ainsi 33 langues sont disponibles à la synthèse.

16 Le système Festival (Black et Taylor, 1997) a été développé par CSTR (Centre for Speech Technology Research) développé à l'Université d'Edinbourg en coopération avec ATR, Japon. Le système est disponible pour l'anglais, l'espagnol et le gallois. MBROLA a été développé pour des applications multilingues non-commerciales par (Dutoit et Leich 1993 ; Dutoit et al., 1996) et lancé par le laboratoire TCTS de la Faculté Polytechnique de Mons, Belgique. Enfin Whistler (Windows Highly Intelligent STochastic taLkER) est un moteur de synthèse de la parole développé par Microsoft, et fonctionne désormais sous Windows 2000, Windows XP, et Microsoft Phone. Un texte tapé à partir du clavier peut être ainsi immédiatement «lu » par l'ordinateur. 
17 Les systèmes ont fait de gros progrès en quelques décennies, améliorant par exemple considérablement l'aspect de l'intelligibilité au niveau phonétique et phrastique, ou en introduisant comme chez Orange Labs par exemple, des éléments paralinguistiques tels que des rires, des hésitations (Ségalen et al., 2008). Les systèmes par " concaténation " ont ces dernières années, renouvelé leurs méthodes. Outre les systèmes "traditionnels", d'autres systèmes dits «à base de corpus " permettent de stocker dans la base de données, moyennant l'augmentation de la mémoire nécessaire, des diphones ${ }^{3}$ identiques (ou autres segments de taille différente) prélevés dans le corpus avec leurs différents contextes prosodiques.

18 Certes les systèmes s'approchent de la qualité naturelle en supprimant les discontinuités, mais une question reste posée concernant les patrons mélodiques, et le rapport de ces patrons au sens et à la subjectivité. C'est le but de cette communication que d'apporter ces nouveaux éléments de nature linguistique pour améliorer les systèmes de synthèse.

\section{Corpus, banque de données et mélismes}

\subsection{Codage, corpus, banque de données}

19 Ce travail repose sur l'observation fine des réalisations des proéminences mélodiques réalisées dans des conditions d'expression subjective, et plus précisément dans un contexte de parole où la personne est amenée à s'impliquer naturellement dans son discours, par le récit par exemple de souvenirs marquants de sa vie. On ne doute plus aujourd'hui que l'une des fonctions de la proéminence mélodique et prosodique des mots soit de nature expressive et subjective. Dans le cadre du mot mélodiquement proéminent (un ou au plus deux mots successifs), on observe ainsi de forts contrastes mélodiques et/ou usage des valeurs mélodiques les plus élevées du registre du locuteur, ce qui se traduit par des pentes mélodiques montantes ou descendantes plus abruptes, accompagnées souvent de ralentissement du débit, de pics d'intensité, tout ceci entraînant un effort plus soutenu de la part du locuteur. Comme précisé ci-dessus, les proéminences mélodiques réalisées dans le contexte de l'unité lexicale (voire grammaticale) en de telles conditions affectives ont été appelées « mélismes » (CaelenHaumont et Bel, 2000).

Sachant que l'analyse des mélismes requiert une certaine finesse technique pour pouvoir évaluer les modulations, nous avons opté pour une précision double de la précision traditionnelle qui partage généralement le registre mélodique en 4 niveaux. Nous découpons donc le registre en 8 niveaux ou plutôt en 16 demi-niveaux mais en procédant de telle sorte que nous regroupons deux à deux des demi-niveaux entre le $2 \mathrm{e}$ et le 15e, laissant en fait 2 demi-niveaux pour les valeurs extrêmes, les plus aiguës (/A/) et les plus graves $(/ \mathrm{g} /)$. Il est plus exact de dire que ces niveaux extrêmes se comportent comme des seuils pour respectivement les valeurs au-delà de /S/ (soit les plus aiguës qui alors sont codées /A/) ou en-deçà de /i/ (inversement les plus graves, codées $/ g / /$, afin d'intégrer ces valeurs aiguës et quelques autres excentrées du registre. Nous obtenons donc au total 9 degrés : grave (g), infra (i), bas (b), central (c), moyen (m), élevé (e), Haut (H), Supra (S), Aigu (A).

21 A titre d'illustration des codages, la Figure 1 ci-dessous présente un extrait de deux mélismes distincts (locutrice LR2, la plus jeune). Le mélisme aux valeurs les plus aiguës 
a été sélectionné et apparaît en grisé. La fenêtre supérieure montre la courbe de F0 issue du fichier Manipulations de Praat (Boersma et Weenink, 2012), l'inférieure, le TextGrid avec ses différentes tires ${ }^{4}$ : en haut tire 1, la segmentation et l'étiquetage manuels des différents mots, puis de bas en haut les tires issues des calculs automatiques, en bas les valeurs de fréquence fondamentale ( $\mathrm{FO}$ ) en $\mathrm{Hz}$, leur codage en demi-tons (ST), puis leur codage alphabétique avec double passage pour mettre en capitales les valeurs aiguës identifiant un mélisme, et enfin sous la tire de segmentation, les « syllabes mélodiques ». Ces syllabes mélodiques, désignées sous cette appellation par simple analogie structurelle avec les syllabes linguistiques, correspondent à l'espace temporel compris entre deux cibles mélodiques successives et calculées de manière automatique.

Figure 1 : Extrait de parole "... des moules, des huîtres un (truc comme ça) », Locutrice LR2 sous Praat

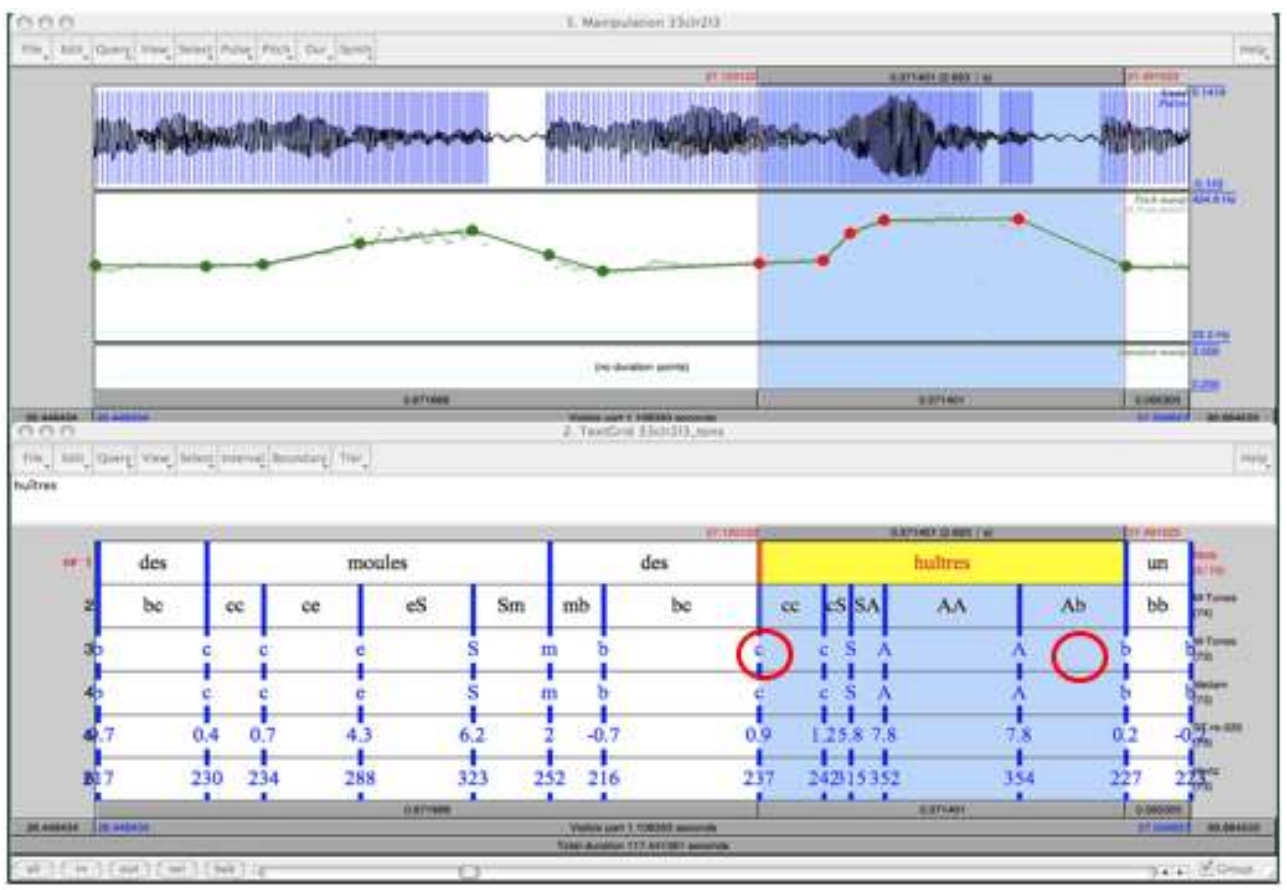

De haut en bas, on distingue la courbe de F0 du fichier Manipulations, et dessous le TextGrid comportant différentes « tires » ou lignes d'étiquetage : au niveau supérieur, celle de la segmentation et étiquetage manuel en mots, puis de bas en haut, les tires calculées de manière entièrement automatique. Les cercles entourant les cibles /c/ et /b/ sont les points de contact entre la courbe intonative et la structure du mélisme (appelées ci-dessous respectivement Pied de l'Amorce ou Ap, et Pied de la Coda ou CP).

Les corpus sur lesquels se fonde cette étude, ont été enregistrés selon le protocole PFC (Phonologie du Français Contemporain, http://www.projet-pfc.net/, Durand et al., 2005). Parmi les 16 locuteurs enregistrés (32 heures environ) dans le Médoc (Cussac Fort-Médoc, dans le vignoble du «Haut-Médoc » à une quarantaine de km au nord de Bordeaux), nous avons sélectionné une famille de 4 locutrices, remarquable en terme de nombre de générations ( 4 au total, soit de l'arrière grand-mère à l'arrière petitefille, HV1, SP1, LR1, LR2), de stabilité géographique (même village, même maison, mêmes locaux depuis l'origine), et professionnelle (viticulture). Les corpus comprenaient outre des lectures obligées, des discours libres sur la base de dialogues entre personnes de la même famille, et de discours avec l'expérimentateur qui posait 
des questions sur la vie d'autrefois, les souvenirs d'enfance ou familiaux marquants, les conditions de vie d'hier et d'aujourd'hui.

Dans ces huit heures d'enregistrement de conversations spontanées, les mélismes ont été identifiés de manière entièrement automatique. Ainsi le corpus, segmenté ou non, est soumis à la procédure MISTRAL+ (Weber et al., 2012 pour la dernière version). En quasi-temps réel, le corpus est entièrement annoté en termes de cibles mélodiques. Conformément à ce qui sera précisé ci-dessous au paragraphe 4.1.1., il suffit alors de repérer les cibles du corpus mélodiquement les plus hautes, à savoir contenant $\mathrm{H}, \mathrm{S}$ ou A (niveaux $7,8,9)$ pour identifier un mot mélismé dans son contexte.

Désormais la procédure MISTRAL+ permet de créer automatiquement un fichier excel rassemblant toutes les tires manuelles et automatiques du fichier Praat-TextGrid, avec la possibilité d'utiliser les codes API.

Ces contextes porteurs de mélismes ont donc été consignés dans une vaste banque de données comportant toutes les informations prosodiques nécessaires. La Figure 2 cidessous présente un extrait de cette banque de données :

Figure 2. Extrait de la banque de données, locutrice HV1.

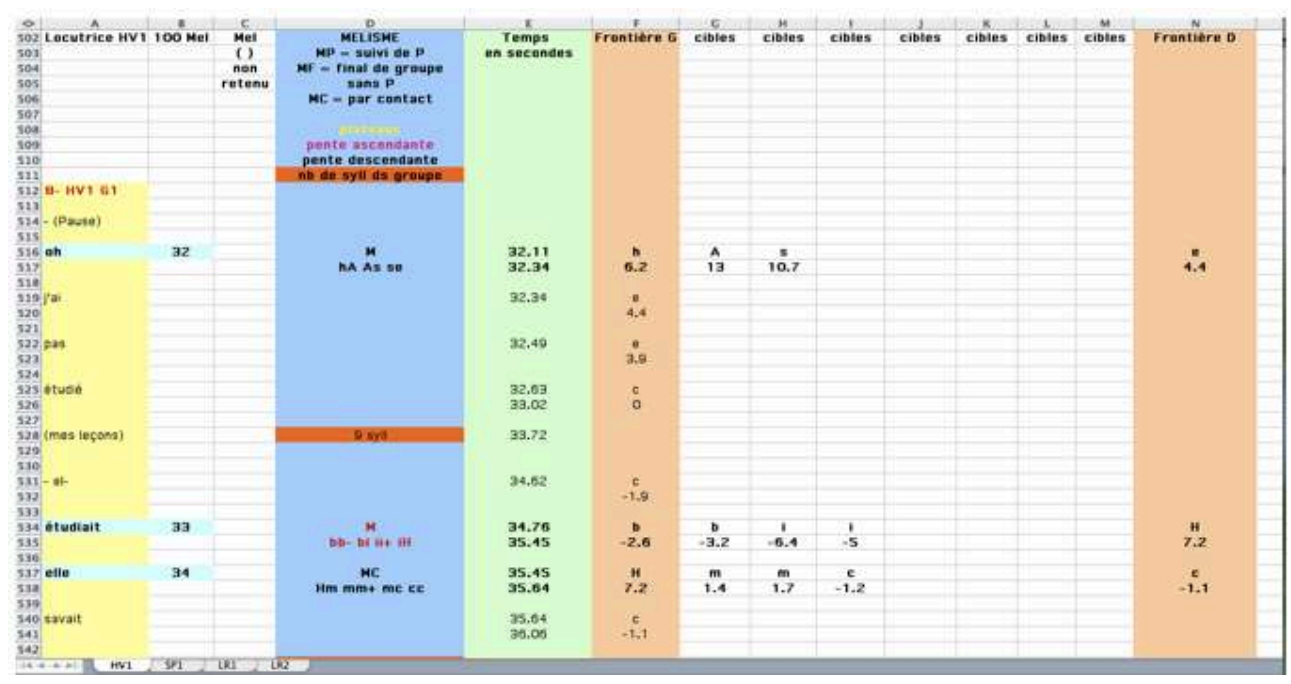

- la colonne A à gauche porte le nom du fichier de parole de la locutrice, et sous celui-ci la liste des unités linguistiques avec les pauses. Les lignes en gras (mots et valeurs) correspondent aux mots mélismés,

- la colonne B indique le numéro d'ordre du mélisme (sur 100 par locutrice),

- la colonne $\mathrm{C}$, la liste des mélismes éventuellement non retenus,

- la colonne $\mathrm{D}$ porte plusieurs informations, à savoir le type de mélisme :

- final de groupe devant pause ou MP,

- final de groupe sans pause ou $M F$,

- interne au groupe ou $M$,

- par contact ou MC.

Toujours colonne D, sous la ligne indiquant le type de mélisme, figure la structure du mélisme définie en termes de "syllabes mélodiques". Par exemple pour le mélisme $\mathrm{n}^{\circ} 32$ ci-dessous /oh/ et ses 4 cibles mélodiques, /h, A, s, e/, on obtient les 3 syllabes mélodiques $/ \mathrm{hA}$, As, se/.Toujours relativement à cette colonne, et attachée au type de mélisme et sa structure syllabique mélodique, une couleur différente permet 
d'identifier les mélismes de pente globale ascendante (graphie rouge, cf mélisme $\mathrm{n}^{\circ} 33$ ), descendante (en noir, cf mélisme $\mathrm{n}^{\circ} 32$ ), et ceux qui sont réalisés en plateau (en jaune). En fait comme ces mélismes en plateau sont très rares, ils ont tous été rattachés en fonction de leur configuration, aux mélismes de pente ascendante (les plus nombreux). Cette colonne mentionne aussi en encadré orange le nombre de syllabes du groupe entre 2 pauses.

- la colonne E mentionne l'adresse temporelle (début / fin de l'unité) en secondes avec une précision de l'ordre de la centiseconde,

- les colonnes suivantes de $\mathrm{F}$ à $\mathrm{N}$ indiquent le niveau mélodique (9 niveaux possibles) de la cible mélodique en codage alphabétique (cf le détail au paragraphe suivant) et dessous, le codage exprimé en demi-tons (positif ou négatif comme il se doit autour de la valeur moyenne de niveau 0 ). Les première $(\mathrm{F})$ et dernière colonnes $(\mathrm{N})$ indiquent respectivement les valeurs aux frontières gauche et droite du mélisme.

Cette présentation d'ensemble faite, nous nous attachons maintenant à décrire les principales étapes dans la construction des proéminences subjectives, d'abord au niveau prosodique, et ensuite minimalement au niveau linguistique. Le paragraphe 4 et les suivants proposent donc la description de la méthode à appliquer en génération de la parole.

\section{Analyse prosodique}

Nous nous intéressons maintenant aux propriétés internes des mélismes, c'est-à-dire à la structure même du mélisme sous l'angle tout d'abord de ses « syllabes mélodiques ».

\subsection{Analyse mélodique}

\subsubsection{Structurer le registre du locuteur}

Notre étude est précise, et comme nous l'avons déjà dit, nous avons divisé le registre des locuteurs en 9 niveaux, soit du plus aigu au plus grave : Aigu (A), Supra (S), Haut $(\mathrm{H})$, élevé (e), moyen $(\mathrm{m})$, central (c), bas (b), infra (i), grave (g). Les niveaux les plus aïgus sont écrits en capitales.

En prosodie, les recherches sur la proéminence (qu'elle soit de nature syntaxique, sémantique ou pragmatique) ont massivement montré depuis plusieurs décades que les niveaux mélodiquement les plus hauts étaient impliqués dans ce mécanisme de perception d'une mise en relief.

31 Les travaux présentés ici sont dans le prolongement de ces études. Le registre des locuteurs étant divisé en 9 , les niveaux les plus hauts 8 et 9 sont à l'évidence concernés. Le niveau 7 appartenant au tiers du registre le plus élevé l'est certainement aussi. Comme le démontrent (se reporter à l'annexe) les 7 exemples sonores sur les 8 de démonstration fournis au total dans le cadre de cette communication (fichiers sons $\mathrm{n}^{\circ}$ 2, 4, 6, 8 et 10 les deux premiers mélismes), une cible de hauteur $\mathrm{H}$ est suffisante pour être perçue comme une proéminence mélodique, quelle que soit la locutrice.

Ce qui importe en fait c'est le contraste avec le contexte de l'énoncé et notamment le contexte antérieur: si le registre est assez grave globalement ou localement, $\mathrm{H}$ est pertinent. Inversement si le registre antérieur ou global est assez haut, le niveau $\mathrm{H}$ risquera de ne pas pouvoir marquer une proéminence : c'est pour cela qu'une condition 
supplémentaire a été imposée, à savoir qu'il existe un contraste avec le contexte précédent d'au moins 3 niveaux. Dans un registre global haut, un mélisme pourra alors être produit avec un fort contraste interne, alliant un niveau plus grave que le niveau antérieur ou global (ex : un niveau /b, i, g/) et un niveau /S/ ou /A/. Dans notre étude, les 3 degrés les plus aigus (A, $\mathrm{S}, \mathrm{H})$, détectés automatiquement par la procédure, signent donc l'existence d'un mélisme.

La procédure MISTRAL+ (Caelen-Haumont et Auran, 2004 ; Weber et al., 2012) ajoute automatiquement dans une tire correspondante une cible entre les frontières des unités lorsqu'elle reconnaît au niveau du nouveau échantillon de parole (distant de 10 ms du précédent) une valeur mélodique supérieure à un seuil de variation $(3 \%)$ par rapport à celle du précédent. Nous obtenons donc dans les TextGrids une suite de cibles mélodiques, exprimées en $\mathrm{Hz}$, converties en demi-tons en fonction du F0 moyen, minimum et maximum du locuteur, et codées alphabétiquement ${ }^{5}$, comme nous l'avons vu au début du paragraphe.

Comme nous l'avons vu, entre deux cibles successives, l'espace mélodique constitue une « syllabe mélodique ». Dans notre corpus, le nombre de ces syllabes mélodiques dans les mélismes s'élèvent à 9 syllabes au plus.

\subsubsection{Structurer le mélisme en parties et sous-parties}

Malgré l'extraordinaire variabilité des mélismes et de leur structure interne, nous avons pu repérer les parties constituantes, les unes facultatives, les autres obligatoires autrement dit, définitoires (cf Tableau 1 ci-dessous).

$\mathrm{Au}$ minimum un mélisme se compose de deux cibles - soit une seule syllabe mélodique-, formant le noyau, partie essentielle du mélisme, toujours présente car définitoire.

37 Ces cibles exercent alors deux fonctions, l'une sur le plan de l'intonation, une fonction démarcative, aux bornes du mélisme (respectivement pour la cible initiale, pied de l'amorce ou $A p$ et pour la cible finale, pied de la coda ou $C p$ ), et l'autre, sur le plan de l'unité lexicale ou grammaticale, une fonction subjective.

Cette fonction subjective attachée à la partie définitoire du mélisme, la plus contrastive, est assurée par le Noyau et ses frontières, soit le pied ou $N p$ et par sa tête ou Nt. Bien entendu au-delà de 2 syllabes mélodiques, fonctions démarcative et subjective ont moins de chance de se confondre. Il arrive d'ailleurs régulièrement que les bornes du mélisme, $A p$ et/ou $C p$, ne soient pas présentes, au contraire du corps du noyau, $N c$, qui est présent le plus souvent. 
Tableau 1. Structure des mélismes : parties et sous-parties, population de chacune d'elles, propriétés et fonctions.

\begin{tabular}{|c|c|c|c|}
\hline \multirow{2}{*}{$\begin{array}{c}\text { Parties } \\
\text { principales }\end{array}$} & \multirow{2}{*}{$\begin{array}{c}\text { Population } \\
\text { et } \\
\text { pourcentages }\end{array}$} & \multicolumn{2}{|l|}{ Sous-parties } \\
\hline & & Définition & Fonction \\
\hline \multirow{2}{*}{$\begin{array}{c}208 \\
\text { AMORCE } \\
(=A)\end{array}$} & $208(52 \%)$ & $\begin{array}{l}\text { Ap: pied de l'amorce } \\
\text { Cible unique } \\
\text { Optionnel }\end{array}$ & $\begin{array}{l}\text { Intonation, } \\
\text { fonction } \\
\text { démarcative }\end{array}$ \\
\hline & $46(12 \%)$ & $\begin{array}{l}\text { Ac: corps de l'amorce } \\
\text { Zéro, une ou plusieurs cibles } \\
\text { Optionnel et souvent absent }\end{array}$ & $\begin{array}{l}\text { Mélisme, } \\
\text { Fonction } \\
\text { subjective }\end{array}$ \\
\hline \multirow{3}{*}{$\begin{array}{c}400 \text { NOYAU } \\
(=N)\end{array}$} & $400(100 \%)$ & $\begin{array}{c}\text { Np : pied du noyau } \\
\text { Cible unique } \\
\text { Obligatoire }\end{array}$ & $\begin{array}{l}\text { Mélisme, } \\
\text { Fonction } \\
\text { subjective }\end{array}$ \\
\hline & $286(72 \%)$ & $\begin{array}{l}\text { Nc : corps du noyau } \\
\text { Zéro, une ou plusieurs cibles } \\
\text { Optionnel et souvent présent }\end{array}$ & $\begin{array}{l}\text { Mélisme, } \\
\text { Fonction } \\
\text { subjective }\end{array}$ \\
\hline & $400(100 \%)$ & $\begin{array}{l}\text { Nt : tête du noyau } \\
\text { Cible unique } \\
\text { Obligatoire }\end{array}$ & $\begin{array}{l}\text { Mélisme, } \\
\text { Fonction } \\
\text { subjective }\end{array}$ \\
\hline \multirow{2}{*}{$\begin{array}{c}259 \text { CODA } \\
(=\mathrm{C})\end{array}$} & $61(15 \%)$ & $\begin{array}{c}\text { Cc: Corps de la coda } \\
\text { Zéro, une ou plusieurs cibles } \\
\text { Optionnel et souvent absent }\end{array}$ & $\begin{array}{l}\text { Mélisme, } \\
\text { Fonction } \\
\text { subjective }\end{array}$ \\
\hline & $259(65 \%)$ & $\begin{array}{l}\text { Cp : pied de la Coda } \\
\text { Cible unique } \\
\text { Optionnel }\end{array}$ & $\begin{array}{l}\text { Intonation, } \\
\text { fonction } \\
\text { démarcative }\end{array}$ \\
\hline
\end{tabular}

Toutes structures confondues, les mélismes (cf Tableau 1 ci-dessus) se composent généralement - et au plus - de 3 parties, à savoir :

- l'Amorce (A), elle-même décomposable en pied $^{6}(\mathrm{Ap})$ comme on vient de le lire, et en corps (Ac),

- le Noyau ( $N$ ), qui se divise en pied (Np), en corps $(N c)$ et en tête (Nt),

- la Coda $(C)$ se divisant quant à elle, en corps $(C c)$ et en pied $(C p)$.

40 Nous retrouvons d'ailleurs ici le modèle courant rencontré dans les processus phonétiques (à base physiologique) tels que les voyelles ou les syllabes: une phase initiale de transition entre deux processus, une phase médiane de stabilité qui possède les propriétés du phénomène les mieux établies, et une phase terminale de nouvelle transition vers le processus suivant.

Concernant un point méthodologique, par souci non seulement d'exactitude mais aussi de reproductibilité dans le temps, nous précisons que lorsque les cibles, adjacentes ou non, étaient de même valeur mélodique en codage alphabétique (ex : / $\mathbf{S ~ S ~ H ~ b ~ e / ~ o u ~ / H ~}$ $\mathbf{H}$ e $\mathrm{m} \mathrm{H}$ /, etc.), nous nous sommes reportée aux valeurs numériques, une valeur plus basse ou plus haute permettant d'affecter une cible à telle ou telle sous-partie sans avoir à l'interpréter. Dans le dictionnaire des mélismes (cf extrait en annexe, Tableau 11), les cibles de même codage alphabétique ayant des valeurs mélodiques différentes sont notées avec une minuscule pour la valeur la plus basse (ex : /ib bH $\mathbf{H h} /$ ).

Plus précisément, parties et sous-parties excercent donc des fonctions différentes (Caelen-Haumont, 2009b). Les bornes (Ap et Cp) du mélisme exercent une fonction intonative (fonction démarcative et de mise en relation des mélismes avec les autres unités du discours), les bornes du noyau ( $\mathrm{Np}$ et $\mathrm{Nt}$ ) se situent sur le plan subjectif (appropriation du discours), par leurs fonctions démarcative et discriminative (faire 
entendre et faire comprendre la proéminence), et élective (faire-croire en partageant sa croyance, voir Caelen-Haumont, 2009a).

Quant au «corps» des différentes parties (Ac, Nc et Cc), leur fonction est essentiellement discriminative (renforcer et étoffer la perception de la proéminence) et esthétique (modulation mélodique, exploitée notamment dans le chant). En ce qui concerne notamment les nombreux $N c$, ils exercent une fonction de renforcement et de soutien mélodique (sans doute aussi physiologique) des parties centrales du Noyau les plus extrêmes et définitoires, en mettant en relief la valeur significative.

Tableau 2

\begin{tabular}{|c|c|c|c|c|c|c|c|c|c|c|c|}
\hline \multirow{7}{*}{$\begin{array}{c}4 \\
\text { cibles }\end{array}$} & $\begin{array}{c}\text { Pied de } \\
\text { l'Amorce } \\
\text { Ap }\end{array}$ & $\begin{array}{c}\text { Corps de } \\
\text { I'Amorce } \\
\text { Ac }\end{array}$ & \begin{tabular}{|c|} 
Pied \\
du \\
Noyau \\
Nip \\
\end{tabular} & \begin{tabular}{|c|} 
Corps \\
du \\
Noyau \\
Ne \\
\end{tabular} & \begin{tabular}{|c|} 
Tête \\
du \\
Noyau \\
Nt
\end{tabular} & $\begin{array}{c}\text { Corps } \\
\text { de la } \\
\text { Coda } \\
\text { Ce }\end{array}$ & $\begin{array}{c}\text { Pied de } \\
\text { la Coda } \\
\text { Cp }\end{array}$ & $\begin{array}{c}\text { Mélismes } \\
\text { Croissants }\end{array}$ & $\begin{array}{c}\text { Mélismes } \\
\text { Décroissants }\end{array}$ & \begin{tabular}{c|} 
Mélismes \\
Croissants et \\
Décroissants
\end{tabular} & $\begin{array}{c}\text { Total } \\
\%\end{array}$ \\
\hline & $\bullet$ & $\bullet$ & $\bullet$ & & $\bullet$ & & & 4 & 1 & 5 & 4 \\
\hline & $\bullet$ & & $\bullet$ & $\bullet$ & $\bullet$ & & & 20 & 5 & 25 & 19 \\
\hline & $\bullet$ & & $\bullet$ & & $\bullet$ & & $\bullet$ & 31 & 3 & 34 & 25 \\
\hline & & & $\bullet$ & $\bullet$ & $\bullet$ & & & 15 & 7 & 22 & 16 \\
\hline & & & $\bullet$ & $\bullet$ & $\bullet$ & & $\bullet$ & 36 & 8 & 44 & 33 \\
\hline & & & $\bullet$ & & $\bullet$ & $\bullet$ & $\bullet$ & 4 & 1 & 5 & 4 \\
\hline \begin{tabular}{|l|} 
Total \\
\end{tabular} & & & & & & & & 110 & 25 & 135 & $101 \%$ \\
\hline \multirow{10}{*}{$\begin{array}{c}5 \\
\text { cibles }\end{array}$} & $\bullet$ & $\bullet$ & $\bullet$ & & • & & & 1 & 0 & 1 & 1 \\
\hline & $\bullet$ & $\bullet$ & $\bullet$ & $\bullet$ & $\bullet$ & & & 7 & 1 & 8 & 5 \\
\hline & $\bullet$ & & $\bullet$ & $\bullet$ & $\bullet$ & & & 12 & 4 & 16 & 11 \\
\hline & $\bullet$ & $\bullet$ & $\bullet$ & & $\bullet$ & & $\bullet$ & 9 & 0 & 9 & 6 \\
\hline & $\bullet$ & & $\bullet$ & $\bullet$ & $\bullet$ & & $\bullet$ & 37 & 5 & 42 & 29 \\
\hline & - & & - & & $\bullet$ & $\bullet$ & $\bullet$ & 13 & 2 & 15 & 10 \\
\hline & & & $\bullet$ & $\bullet \bullet$ & $\bullet$ & & & 11 & 0 & 11 & 7 \\
\hline & & & $\bullet$ & $\bullet$ & $\bullet$ & & $\bullet$ & 27 & 1 & 28 & 19 \\
\hline & & & $\bullet$ & $\bullet$ & $\bullet$ & $\bullet$ & $\bullet$ & 13 & 3 & 16 & 11 \\
\hline & & & • & & - & $\bullet \bullet$ & • & 1 & 0 & 1 & 1 \\
\hline \begin{tabular}{|l|} 
Total \\
\end{tabular} & & & & & & & & 131 & 16 & 147 & $100 \%$ \\
\hline
\end{tabular}

Les différents patrons les plus fréquents réalisés par les 4 locutrices (symbolisés par une suite de différents points 頤 dans les structures des mélismes de 4 et 5 cibles mélodiques, et populations en fonction des mélismes croissants $C$, décroissants $D$, de leur total $C$ et $D$, et pourcentages. Les majuscules $A, N$ et $C$ dans les en-têtes de colonnes correspondent aux 3 parties possibles du mélisme et signifient respectivement (Amorce en gris clair, Noyau en gris moyen, et Coda du mélisme en gris clair), et les minuscules, leurs sous-parties éventuelles, à savoir $p, c, t$, pied (ou début), corps (partie centrale) pour chacune de ces parties et tête ( $t$ indiquant l'extrémité du Noyau du mélisme, le plus souvent la cible la plus haute, n'appartient qu'au Noyau).

Par ailleurs leurs effectifs sont très différents. Dans notre banque de données les pieds de la Coda $(C p)$ sont souvent présents $(65 \%)$, et en plus grand nombre que les pieds de l'Amorce (Ap, $52 \%$ ). En ce qui concerne les corps, les effectifs sont extrêmement variables puisqu'ils sont très nombreux dans le corps du Noyau (Nc, $72 \%)$, rares dans la Coda ( $C c, 15 \%)$, et encore plus rares dans le corps de l'Amorce (Ac, $12 \%)$.

Pour nous concentrer sur l'essentiel, nous avons recensé les structures les plus nombreuses, à savoir les structures à 3 et 4 syllabes mélodiques (soit 4 et 5 cibles), représentant au total 282 unités ( $71 \%$ ) sur 400 . Précisons tout d'abord que toutes les combinaisons ne sont pas possibles: par exemple, les structures ne peuvent pas commencer par $A c$ ni se terminer par $\mathcal{C}$, car ces deux cibles nécessitent la présence respectivement de $A p$ et de $C p$. Mais par ailleurs, plusieurs cibles peuvent être réalisées 
dans une sous-partie, par exemple Tableau 2 ci-dessus, ligne 15, la structure / $N p-N c N c$ $N c-N t /$, dans laquelle 3 cibles composent le corps du Noyau $N c$, toutes par nature contrastives.

Dans le cas de 3 syllabes mélodiques, 6 structures en tout se répartissent les 135 mélismes croissants et décroissants, et pour les 4 syllabes mélodiques, les 147 mélismes sont par ailleurs ventilés en 10 structures.

La constatation d'ensemble est certes qu'il existe une certaine diversité : les locuteurs varient les formes dans une certaine mesure, mais tout en privilégiant toutefois certaines d'entre elles. Il est tout à fait intéressant de noter par exemple que lorsque l'on passe de 3 à 4 syllabes mélodiques (c'est-à-dire de 4 à 5 cibles), la seule différence qui existe entre les structures les plus usitées, à savoir de $/ A p N p N t C p /$ aux autres c'està-dire les 5 cibles, c'est l'ajout d'un corps soit à l'amorce $(/ A c /)$, soit à la coda $(/ C c /)$, mais surtout au Noyau $(/ \mathrm{Nc} /)$. De même pour les nombreuses structures $/ \mathrm{Np} N c \mathrm{Nt} \mathrm{Cp} /$, c'est aussi l'ajout d'un corps, soit à la Coda $(/ \mathrm{Cc} /)$, mais surtout encore au noyau $(/ \mathrm{Nc} /)$. Renforcer les structures, c'est donc étoffer au sens propre en "donnant du corps ».

Les structures les plus courantes sont donc:

- /Ap Np Nt $C p /$ pour 4 cibles, et /Ap Np Nc Nt $C p /$ pour 5 cibles,

- $/ N p N c N t C p /$ pour 4 cibles, et / $N p N c$ Nc Nt $C p /$ pour 5 cibles.

Dans le cadre de la génération de parole du français, la dizaine de structures les plus nombreuses exposée au Tableau 2 ci-dessus, peut fournir des patrons de combinaison des cibles à la fois adéquats et diversifiés. Et cette variabilité et cette diversification, c'est précisément ce qui fait encore défaut à la synthèse.

\subsubsection{Choisir un patron mélodique croissant ou décroissant (C, D, P)}

49 Trois types de pentes sont possibles, les croissantes (C), décroissantes (D) et plateaux (P) qui par définition ne devraient pas avoir de pente.

Précisons tout d'abord qu'il s'agit de pente globale, car généralement le mélisme qu'il soit montant ou descendant, est constitué de 2 pentes, l'une plus longue que l'autre en termes de nombre de cibles. Cette partie plus développée est souvent flanquée des valeurs opposées extrêmes, soit les plus haute et plus basse de tout le mélisme. A moins d'un mélisme constitué seulement par 2 cibles, ce ne sont pas les valeurs aux bornes du mélisme qui décident de la forme de la pente, car celles-ci sont généralement dépassées par les valeurs extrêmes situées dans le noyau. Ces valeurs aux frontières sont d'ailleurs plus liées à l'intonation du groupe ou de la phrase qu'au mélisme lui-même. Selon donc que la cible la plus basse du mélisme précède ou suive la plus haute, le mélisme sera considéré globalement comme montant ou descendant.

51 En fait la stabilité pure étant extrêmement rare, on peut dans la très grande majorité des cas rattacher le plateau à l'une des deux pentes. Ainsi parmi les 400 mélismes, nous n'avons relevé finalement que 5 mélismes en plateau, que nous avons finalement tous reclassés en croissants du fait que le nombre de leurs valeurs était plus grand dans la partie montante (en gras dans l'exemple qui suit, locutrice HV1, mélisme $\mathrm{n}^{\circ} 158$ <oui>, de type $\mathrm{M}$ (mélisme interne au groupe), structure /hh hS Sh/).

Deux mélismes ont été inclassables, du fait que leurs valeurs fréquentielles étaient totalement identiques, que les valeurs avant et après le niveau maximum étaient aussi identiques, et que la valeur la plus basse du contraste majeur central était aussi identique à la valeur finale. Comme cela était de toute manière indécidable, et donc 
sans conséquence pour le choix, nous les avons comptés parmi les mélismes les plus nombreux, à pente croissante. Ainsi dans notre corpus, nous comptons 329 mélismes ascendants et 71 descendants : les ascendants sont ainsi 4,6 fois plus nombreux.

Parmi les 71 descendants, on s'aperçoit que les mélismes par contact (MC) sont beaucoup plus nombreux ( $39 \%$ ) que chez les croissants ( 1 seule occurrence, $0.3 \%$ ). Or les $\mathrm{MC}$ sont tous à une seule exception près, des mots grammaticaux (prépositions, conjonctions, présentatifs, déterminants, pronoms). Par ailleurs si l'on regarde les types de mélismes décroissants, on trouve aussi une forte proportion de mots grammaticaux (au nombre de 45, soit $63 \%$ ). Restent ensuite 26 mots lexicaux. Si on les analyse, 20 correspondent à des mots en fin de groupe avec ou sans pause, ou en position pénultième ( 5 référencés $M$, soit en position interne). Seuls 4 mots lexicaux $M$ se trouvent donc en position médiane auxquels s'ajoutent $2 \mathrm{MC}$.

On peut donc dire que dans notre corpus, les mélismes décroissants se composent à $92 \%$ de mots grammaticaux et de mots lexicaux en fin de groupe pour lesquels l'intonation impose la pente (mais non le contraste de niveaux et le registre aigu qui identifie de manière indépendante le mélisme).

En génération de parole, les mots grammaticaux et lexicaux en position pénultième et finale de phrase se verront donc plus souvent dotés d'une pente descendante.

\subsubsection{Choisir un prototype mélodique}

Comme on l'a vu ci-dessus, 2 cibles sont requises au minimum (celles du Noyau, $N p$ et $\mathrm{Nt}$ ) pour la constitution d'un mélisme, qui dans ces conditions minimales, fusionne à sa gauche le pied du Noyau (Np) avec le pied de l'amorce (Ap) et à sa droite, la tête du Noyau (Nt) avec le pied de la coda $(\mathrm{Cp})$. Les possibilités en vue de la synthèse concernent donc le choix entre 2 et 7 cibles.

57 La Figure 3 ci-dessous présente le prototype mélodique du mélisme dans notre corpus, calculé pour chacune des cibles avec leur écart-type, à partir des 400 unités, et en fonction des 9 niveaux des cibles. Lorsque nous avions plusieurs cibles dans la même sous-partie, nous avons retenu leur moyenne.

Figure 3

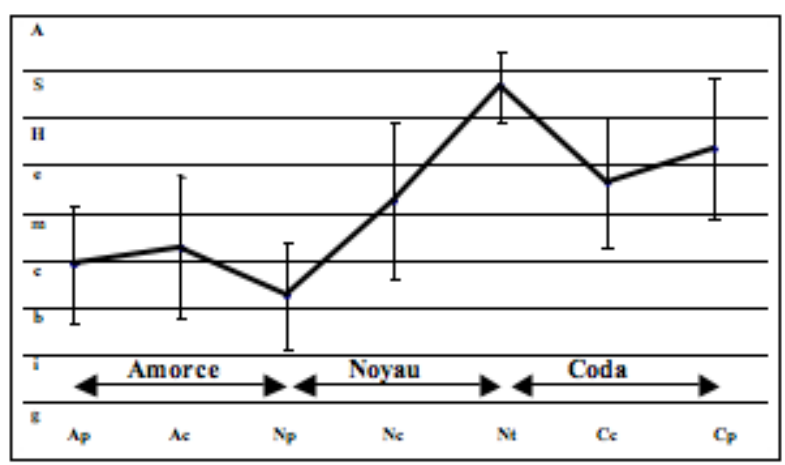

Prototype du mélisme calculé avec la moyenne de chaque cible (Ap, Ac, Np, Nc, Nt, Cc, Cp) et leur écart-type en fonction des 9 niveaux des cibles ( $A, S, H, e, m, C, b, i$ g) à partir des 400 mélismes croissants et décroissants inversés du corpus (4 locutrices)

Dans (Caelen-Haumont, 2009b), nous avons montré que les structures des mélismes décroissants coïncident avec la structure symétriquement inversée des mélismes 
croissants, ce qui nous autorise à mêler les structures quand cela nous paraitt utile. C'est le cas pour la Figure 3 ci-dessus, les prototypes des structures croissantes et décroissantes inversées étant semblables, nous mêlons toutes les structures : seule la valeur des écarts-types a un peu progressé.

Ce prototype englobe les 7 sous-parties potentielles, mais comme nous l'avons vu au paragraphe 4.1.2. ci-dessus, la partie du Noyau $\mathrm{Np} / \mathrm{Nt}$ étant seule obligatoire. Quant aux autres sous-parties, leur présence varie largement (cf ci-dessus, Tableau 1). Bien entendu des prototypes sont aussi disponibles à la synthèse pour les mélismes en fonction du nombre de leurs sous-parties (de 2 à 7), ce qui est l'objet du paragraphe suivant.

\subsubsection{Accorder plus de variabilité à la structure du prototype}

Dans le paragraphe précédent, nous avons présenté la structure maximale de mélisme, réunissant 7 cibles $^{7}$, et donc les conditions de la modulation mélodique la plus étendue. Comme nous l'avons vu au paragraphe 4.1.2., en fait un bon moyen d'accorder une plus grande variabilité aux mélismes est de jouer sur le nombre de cibles. Lorsque le nombre de syllabes mélodiques croît par exemple de 1 cible, passant ainsi de 2 à 3 cibles, puis à 4 , et ce jusqu'à 10 , quelles sont alors les cibles qui sont le plus souvent recrutées?

61 Cette question est intéressante, car elle peut fournir à la synthèse les patrons ou prototypes les plus représentatifs en fonction de nombre de cibles.

Tableau 3

\begin{tabular}{|c|c|c|c|c|c|}
\hline Nombre de cibles & Ap & Ac & Nc & Cc & Cp \\
\hline $\mathbf{3}$ & $17 \%$ & $/$ & $\underline{\mathbf{4 6} \%}$ & $/$ & $38 \%$ \\
\hline $\mathbf{4}$ & $19 \%$ & $1 \%$ & $\underline{\mathbf{5 7} \%}$ & $1 \%$ & $\mathbf{2 2} \%$ \\
\hline $\mathbf{5}$ & $17 \%$ & $5 \%$ & $\underline{\mathbf{5 4} \%}$ & $5 \%$ & $19 \%$ \\
\hline $\mathbf{6}$ & $11 \%$ & $3 \%$ & $\underline{\mathbf{5 3} \%}$ & $15 \%$ & $\mathbf{1 8 \%}$ \\
\hline $\mathbf{7}$ & $11 \%$ & $13 \%$ & $\underline{\mathbf{4 0} \%}$ & $\mathbf{2 0} \%$ & $16 \%$ \\
\hline $\mathbf{8}$ à $\mathbf{1 0}$ & $6 \%$ & $6 \%$ & $\underline{\mathbf{5 5} \%}$ & $\mathbf{2 1 \%}$ & $12 \%$ \\
\hline
\end{tabular}

Evolution des effectifs en pourcentages des cibles mélodiques secondaires (non définitoires) en fonction de leur nombre dans les structures des mélismes

62 Pour répondre à cette question, nous avons comptabilisé ${ }^{8}$ pour chaque mélisme, les cibles qui étaient ajoutées aux cibles définitoires, $N p$ et $N t$. Le Tableau 3 ci-dessus présente ainsi l'évolution des effectifs (en pourcentages) des cibles mélodiques des mélismes lorsqu'on les augmente successivement d'une cible.

Comme on le sait, la structure définitoire présente 1 syllabe mélodique / $\mathrm{Np}-\mathrm{Nt} /$ et donc 2 cibles qui les délimitent. Lorsque l'on augmente successivement d'une cible la structure précédente on passe de 2 cibles à 3 cibles et corolairement de 1 à 2 syllabes. On observe désormais ce qui se passe en dehors des cibles définitoires (toujours présentes).

La première ligne du Tableau 3 ci-dessus montre que la cible la plus souvent utilisée alors ( $46 \%$ et $57 \%$ des cas respectivement pour les 3 et 4 cibles) est $/ \mathrm{Nc} /$. Le patron le plus courant est donc par exemple pour les structures à 3 cibles: / $N p N c N t /$, en ajoutant $/ \mathrm{Nc}$ / aux cibles définitoires $/ \mathrm{Np} /$ et $/ \mathrm{Nt} /$, puis en accord aussi avec le Tableau 2 ci-dessus pour les structures de 4 cibles, $/ \mathrm{Np} N \mathrm{Nc} \mathrm{N} \mathrm{C} / \ldots$ 

dans la sécurité.

\subsubsection{Créer et étoffer les contrastes}

71 La fonction du mélisme étant d'être contrastive, tant de manière externe sur le plan de l'intonation dans la chaîne lexicale que de manière interne en son sein, nous orientons maintenant l'étude vers les moyens de cette mise en contraste.

Comme nous pouvons le constater sur la Figure 3 ci-dessus montrant le prototype, le niveau moyen du contraste mélodique dans le Noyau du mélisme s'étend de la zone basse de /c/ jusqu'à la zone médiane de /S/, soit environ 5 niveaux, ce qui représente la moitié du registre total.

A ce contraste central, s'ajoutent d'autres contrastes tout d'abord de second niveau comme nous l'avons vu au paragraphe précédent, d'une part pour l'amorce entre $A p$ et $N p$, d'autre part pour la coda, entre $N t$ et $C p$, et éventuellement de troisième niveau avec $A c$ dont la valeur contraste à la fois avec les cibles voisines $A p$ et $N p$, et symétriquement avec $C c$ entre $N t$ et $C p$ : le mélisme par définition est une structure éminemment contrastive, et vis-à-vis du contexte antérieur et postérieur intonatif, et vis-à-vis de sa structuration interne. En dehors des cibles frontières de mot ( $\mathrm{Ap}$ et $\mathrm{Cp}$ ) aucune nouvelle cible de valeur supérieure à 圈 $3 \%$ de la précédente, ne peut se surajouter si elle n'est pas contrastive

Corela, $10-1$ | 2012 
$\grave{a}$ gauche comme à droite. En ce sens, les valeurs en plateau (cf par exemple /HH/) divergent donc de plus de 蕰 $3 \%$.

Dans la fonction de l'expression subjective, en production comme en perception, la structure des mélismes repose essentiellement sur deux fonctions: contraster et étoffer, la dernière servant à la première.

S'agissant d'étoffer, c'est-à-dire de créer de la substance mélodique dans le mélisme, des procédés particuliers sont employés. Ces procédés n'ont jamais été mis à jour jusqu'à présent dans le contexte des recherches internationales. Fonction du temps et de l'économie physiologique, ces procédés se fondent sur la répétition des patrons mélodiques, en l'occurrence, des syllabes mélodiques. Ils ne sont pas l'exclusivité des mélismes, ils existent dans le cadre des mots non proéminents, mais c'est bien sûr dans le contexte étendu des mélismes qu'ils sont les plus représentatifs, les mieux mis en relief et qu'ils reçoivent leur expression la plus complète.

Dans le cadre de notre corpus, deux moyens sont utilisés pour étoffer, à savoir, la symétrie et la duplication. Ils concernent les 4 locutrices. Ce phénomène très souvent utilisé est rendu manifeste grâce à la granularité fine de la procédure MISTRAL+. Ainsi en moyenne $69 \%$ (276 unités) des 400 mélismes ont recours à ce procédé, et parmi les locutrices, les scores s'étalent de 62 à $79 \%$. Plus en détail, ces $69 \%$ moyens se ventilent en répétition d'une seule ( $45 \%)$, de deux ( $21 \%)$, ou trois (3\%) syllabe(s) mélodique(s), ce qui donne lieu à 397 répétitions pour ces 276 mélismes. Ceci concerne tous les mélismes, courts, longs, avec une seule syllabe mélodique (ex:/SS/), ou plusieurs. Il va de soi que 2 ou 3 répétitions internes nécessitent des structures mélodiques longues.

\subsubsection{Etoffer par la symétrie}

Concernant tout d'abord la répétition par symétrie, nous pouvons l'illustrer par de maints exemples. Soit le nom propre Junior de structure: $/ \mathrm{mc} \mathrm{cm} \mathrm{mm} \mathrm{mH} \mathrm{Hm} \mathrm{mm/,}$ locutrice HV1. Dans cette séquence, chaque syllabe fait partie du procédé, soit successivement :

$\cdot / \mathrm{mc} \mathrm{cm} /$ est une structure symétrique,

- $/ \mathrm{mm} /$ est la duplication de la même cible mélodique,

- $/ \mathrm{mH} \mathrm{Hm} /$ est à nouveau une structure symétrique,

- $/ \mathrm{mm} /$ est à nouveau la duplication de la même cible mélodique, et qui plus est, la même que précédemment,

- mais plus généralement, / $/ \mathrm{mm} \mathrm{mH} \mathrm{Hm} \mathrm{mm/} \mathrm{est} \mathrm{aussi} \mathrm{une} \mathrm{structure} \mathrm{symétrique} \mathrm{double.}$

Il en résulte que les 7 cibles mélodiques successives se fondent seulement sur 3 cibles différentes $/ m, c, H /$.

Le second exemple utilise le mélisme qui compte le plus de syllabes mélodiques, soit 9 en tout: draconien, /ib bg gb bi im $\mathrm{mm} \mathrm{mH} \mathrm{Hm} \mathrm{mm/,} \mathrm{locutrice} \mathrm{LR1.} \mathrm{Dans} \mathrm{cette} \mathrm{structure,}$

$\cdot / \mathrm{bg} \mathrm{gb} /, / \mathrm{mH} \mathrm{Hm} /$ sont symétriques,

- de plus / $\mathrm{bg} g \mathrm{~g} /$ est inclus dans une autre symétrie : /ib bg gb bi/,

- et de même $/ \mathrm{mH} \mathrm{Hm} /$ dans $/ \mathrm{mm} \mathrm{mH} \mathrm{Hm} \mathrm{mm/,}$

où $/ \mathrm{mm} /$ correspond à la même cible mélodique (et aussi curieusement à la même symétrie que ci-dessus pour Junior, alors que les locutrices sont différentes). Au total, ces 10 cibles mélodiques se réduisent à 6 cibles différentes. 
81 Ces deux exemples illustrent comment les locutrices mettent en valeur les mélismes, faisant varier les cibles dans le temps, augmentant la durée, tout en réduisant en même temps l'effort des tâches de planification et de production grâce à la répétition et la mémoire à court terme.

\subsubsection{Etoffer par la duplication}

Mais ce n'est pas tout. Une étude plus détaillée permet de localiser plus précisément le deuxième procédé, celui de la duplication, en 3 endroits du mélisme :

- au début : on utilise par convention le nom «d'Appel » (ou A) par exemple, locuteur HV1, midi, / $\underline{\mathrm{bb} b \mathrm{~b}} /$,

- à la fin : il reçoit le nom « d'Echo » (ou E), par exemple, locuteur SP1, beau, /bi is SS/,

- entre les deux : il est appelé « Tenue » (ou T), par exemple, locuteur LR1, vrai, /bc cH HS SS Sm/.

Figure 4a.

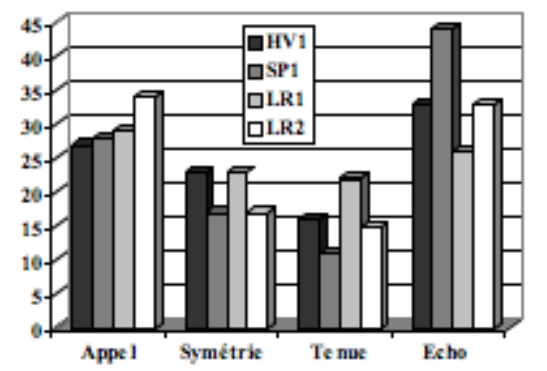

Pourcentages de mélismes par locutrice en fonction des 4 modes de duplication (Appel, Symétrie, Tenue, Echo), et en comptabilisant une, deux et trois duplications (69\%).

Figure $4 b$.

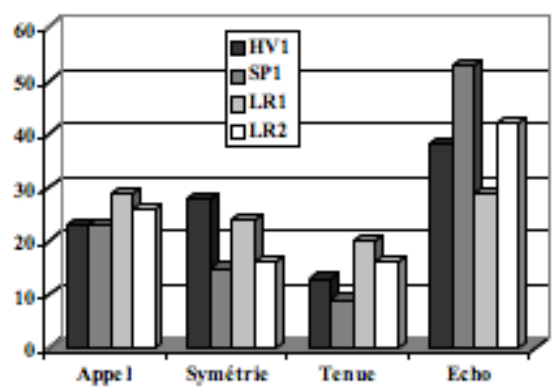

Même chose mais avec une seule duplication (45\%)

Les Figures $4 \mathrm{a}$ et $4 \mathrm{~b}$ ci-dessus présentent les pourcentages des mélismes par locutrice. La Figure 4a montre les taux des pourcentages calculés à propos des 4 sortes de motifs de répétition (Appel, Symétrie, Tenue et Echo), et rassemblant une, deux et trois répétitions des motifs. La Figure $4 \mathrm{~b}$ présente la même chose, mais avec les mélismes les plus nombreux, offrant une seule répétition. 


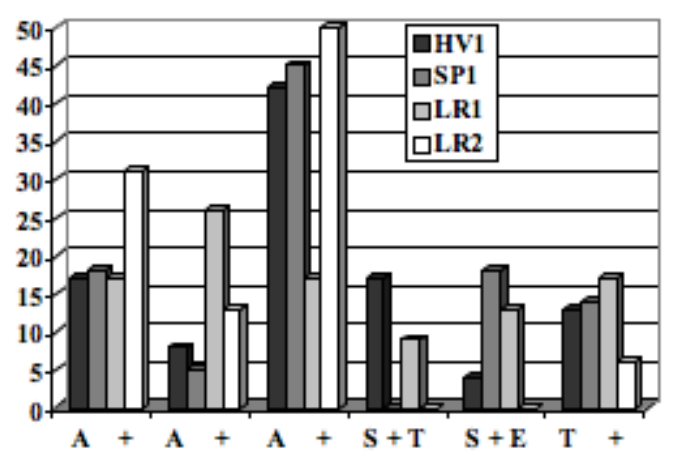

Pourcentages de mélismes par locutrice en fonction des différentes combinaisons des modes de répétition (Appel, Symétrie, Tenue, Echo).

Des Figures $4 \mathrm{a}$ et $4 \mathrm{~b}$, concernant le phénomène de duplication, on constate que l'Appel et l'Echo sont un peu plus fréquents que la Tenue, et sur l'ensemble des 4 phénomènes, supérieurs aussi aux pourcentages de la Symétrie. Dans l'ensemble les scores se ressemblent entre ces 2 figures, mais quand on se restreint à une seule duplication (Figure 4b), l'Echo est encore plus utilisé au détriment de tous les autres, et surtout de l'Appel. Entre les locutrices, on note quelques variations (cf par exemple la locutrice SP1 pour le traitement de l'Echo par rapport aux autres procédés).

La Figure 5 ci-dessus propose les pourcentages des mélismes par locutrice en combinant deux à deux les types de répétition afin de déterminer la combinaison la plus fréquente. Ainsi trois locutrices utilisent le plus souvent la combinaison $\mathrm{A}+\mathrm{E}$ (Appel + Echo). Il est intéressant de constater que la locutrice LR1 s'échappe de ce schéma, pour favoriser a contrario l'Appel combiné avec la Symétrie $(A+S)$ et en second lieu l'Appel avec la Tenue $(A+T)$. C'est d'ailleurs la combinaison $A+S$ qui est la plus résistante après $\mathrm{A}+\mathrm{E}$.

Le Tableau 4 ci-dessous s'intéresse aux effectifs des mélismes offrant 3 motifs de répétition. Bien entendu cela requiert des mélismes aux nombreuses syllabes mélodiques, ce qui est moins fréquent (14 sur 276 ont de 6 à 9 syllabes mélodiques, soit $5 \%$ ), mais par ailleurs ce phénomène concerne les 4 locutrices. Sur les 11 mélismes ayant 3 motifs de répétition, 5 d'entre eux ont de 5 à 9 syllabes. Dans ces conditions, ce tableau vaut surtout pour témoigner de ce processus, en l'interprétant comme une confirmation et une extension de la combinaison des deux répétitions. La combinaison A+S+T (Appel + Symétrie + Tenue) semble être la plus fréquente.

Tableau 4

\begin{tabular}{|c|c|c|c|c|c|}
\hline 3 répétitions & $\mathbf{A}+\mathrm{T}+\mathrm{E}$ & $\mathbf{A}+\mathbf{S}+\mathbf{E}$ & $\mathbf{A}+\mathbf{S}+\mathbf{T}$ & $\mathbf{S}+\mathbf{T}+\mathbf{E}$ & TOTAL \\
\hline HV1 & 0 & 0 & 0 & 1 & 1 \\
\hline SP1 & 1 & 1 & 1 & 1 & 4 \\
\hline LR1 & 0 & 1 & 1 & 1 & 3 \\
\hline LR2 & 1 & 0 & 2 & 0 & 3 \\
\hline TOTAL & 2 & 2 & 4 & 3 & 11 \\
\hline
\end{tabular}

Nombre de mélismes par locutrice offrant une combinaison de 3 répétitions.

A partir de cette étude, se fondant sur les patrons naturels présentés ci-dessus, il est possible en vue de la génération de la parole, de «tisser » des motifs mélodiques variés, 
en s'appuyant sur des bases de composition simple reposant essentiellement sur la répétition des cibles.

Le prochain paragraphe s'intéresse à la structure des mélismes envisagée sous l'aspect de la génération d'arborescences.

\subsubsection{Arborescence et familles : multiplier les structures} fonction d'une part des contrastes plus ou moins importants des cibles extrêmes du Noyau $/ \mathrm{Np} /$ et $/ \mathrm{Nt} /$, et d'autre part en fonction des cibles de leurs frontières externes $(A p$ et $C p$ ) qui varient au gré de l'intonation de groupe et de phrase.

Si les 9 cibles mélodiques partageant le registre du locuteur se combinent toutes à ellesmêmes pour former les syllabes mélodiques, alors il existe au maximum 81 syllabes mélodiques possibles.

On peut ainsi représenter les structures de manière arborescente et les dériver par exemple depuis les cibles externes ( $A p$ et $C p$ ). Ainsi au total on compte 24 familles pour les mélismes décroissants et 37 pour les croissants. $88 \%$ des décroissants se retrouvent d'ailleurs parmi les croissants (en inversant l'ordre des cibles par symétrie pour les rendre compatibles).

Du fait que des structures attestées dans notre corpus composent soit les racines, les nœuds ou les feuilles, il est probable que les nœuds non attestés peuvent néanmoins être des candidats pour la génération de parole.

Ainsi le tableau 5 ci-dessous présente un exemple d'arborescence appartenant à un patron descendant (famille / $\mathrm{Hc} /$ ). Il montre donc comment il est possible de dériver les structures à partir des deux cibles externes du mélisme ( $A p$ et $C p$ ) et qui dans le cadre de cet exemple, sont représentées par les valeurs /H/ (niveau 7 « Haut») et /c/ (niveau 4 , « centré », registre moyen). Par convention ces 2 cibles initiales à partir desquelles on dérive les autres, sont appelées avec leur arborescence générée, une "famille». Ainsi par exemple Figure 1 ci-dessus, le mélisme du mot huitres, comme l'indiquent les 2 cercles rouges, est de la famille /cb/.

Tableau 5 ci-dessous on voit pour la famille / $\mathrm{Hc} /$ quelles sont les structures attestées dans le corpus par ajout successive d'une cible (soulignée), et celles qui ne le sont pas (cases vides). 
Tableau 5

\begin{tabular}{|c|c|c|c|c|c|c|c|c|c|}
\hline \multirow{2}{*}{$N^{\circ}$} & \multirow{2}{*}{$\begin{array}{l}\text { Locu- } \\
\text { trices }\end{array}$} & \multirow{2}{*}{ mots } & \multirow{2}{*}{$\begin{array}{l}\text { Ty- } \\
\text { pes }\end{array}$} & \multicolumn{6}{|c|}{6 niveaux de profondeur au maximum } \\
\hline & & & & 1 & 2 & 3 & 4 & 5 & 6 niveaux \\
\hline \begin{tabular}{|l|}
7 \\
\end{tabular} & HV1 & et & $\mathrm{M}$ & $\mathrm{Hc}$ & & & & & \\
\hline 48 & HV1 & le & M & $\mathrm{Hc}$ & & & & & \\
\hline 136 & SP1 & les & MC & $\mathrm{Hc}$ & & & & & \\
\hline 227 & LR1 & puis & MC & $\mathrm{Hc}$ & & & & & \\
\hline 317 & LR2 & c'est & M & $\mathrm{Hc}$ & & & & & \\
\hline 336 & LR2 & j'étais & MC & & $\mathrm{hH} \mathrm{Hc}$ & & & & \\
\hline 157 & SP1 & bateau & MP & & & He ch he & He ch he $\propto c$ & & \\
\hline 293 & LR1 & c'est & $\mathbf{M}$ & & & $\mathrm{HH} \mathrm{Hm} \mathrm{mc}$ & & & \\
\hline 298 & LR1 & en & M & & & $\mathrm{HH} \mathrm{Hm} \mathrm{mc}$ & & & \\
\hline 51 & HV1 & $\begin{array}{c}\text { cuisi- } \\
\text { nière }\end{array}$ & $\mathrm{M}$ & & & $\mathrm{HH} \mathrm{Hc} \propto \mathrm{c}$ & $\mathrm{Hh} \mathrm{hH} \mathrm{Hc} \propto \mathrm{c}$ & $\mathrm{Hm} \mathrm{mh} \mathrm{hH} \mathrm{Hc} \mathrm{cc}$ & $\mathrm{Hc} \mathrm{cm} \mathrm{mh} \mathrm{hH} \mathrm{He} \mathrm{cc}$ \\
\hline 395 & LR2 & mais & M & & He oc & & & & \\
\hline 20 & HV1 & y'avait & MC & & & He ec oc & & & \\
\hline 283 & LR1 & que & M & & $\mathrm{Hm} \mathrm{mc}$ & & & & \\
\hline 60 & HV1 & elle & MC & & $\mathrm{Hm} \mathrm{mc}$ & & & & \\
\hline 37 & HV1 & $\begin{array}{l}\text { gar } \\
\text { çons }\end{array}$ & $\mathrm{M}$ & & & $\mathrm{Hm} \mathrm{mm} \mathrm{mc}$ & & & \\
\hline 34 & HV1 & elle & MC & & & & $\mathrm{Hm} \mathrm{mm} \mathrm{mc} \mathrm{oc}$ & & \\
\hline
\end{tabular}

Exemple d'arborescence (Famille / $\mathrm{Hc} /$ ).

Mais quelles sont les familles les plus représentées dans notre corpus? Les figures $6 \mathrm{a}$ pour les mélismes croissants et $6 \mathrm{~b}$ pour les décroissants illustrent ce point. Les 3 familles chez les décroissants se retrouvent d'ailleurs chez les croissants (en inversant l'ordre des cibles). Chez les croissants, ce sont les familles avec la cible /b/ à gauche qui sont les plus nombreuses (128 mélismes), et parmi ceux-ci, la famille /bH/ (44 mélismes).

Figure 6a : Les familles les plus nombreuses chez les mélismes croissants

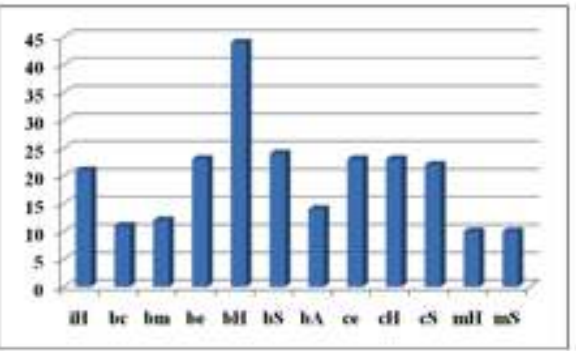

Figure $6 b$ : Les familles les plus nombreuses chez les mélismes décroissants

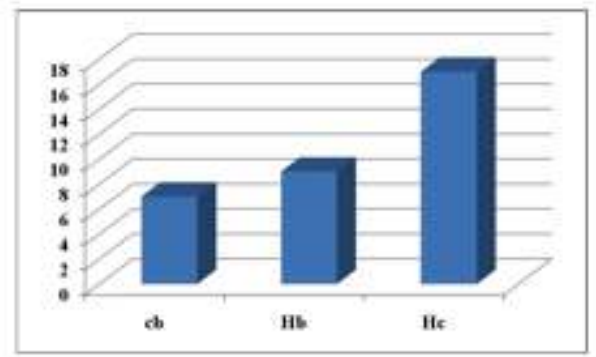


Il est intéressant également d'apporter une information supplémentaire en répertoriant les familles non plus en fonction des bornes du mélisme mais en fonction des bornes du noyau.

La figure 7 ci-dessous montre que parmi les 21 familles qui structurent l'ensemble des noyaux, 7 «familles " (mélismes croissants) sont les plus représentées, à savoir $i H$, is, $b H, b S, b A, c H, c S$. Elles se partagent $82 \%$ des mélismes totaux.

Figure 7. Population de mélismes croissants en fonction des principales familles du noyau.

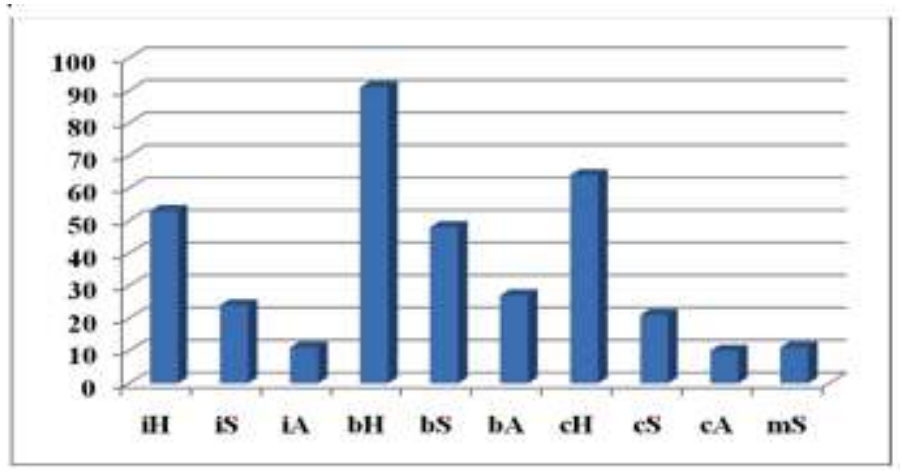

Les mélismes décroissants, quant à eux, au nombre de 71, regroupent 13 familles pour le noyau. Elles se retrouvent toutes dans les 21 familles (toujours en inversant l'ordre des cibles, par effet de symétrie), mais 2 d'entre elles ( $\mathrm{Hb}$ et $\mathrm{Hc}$ ) regroupent $46 \%$ des mélismes décroissants. Un exemple est fourni pour la famille $H c$, déjà présentée Tableau 5 ci-dessus. Pour les mélismes croissants, ces familles figurent aussi parmi les plus nombreuses ( $c H$ et $b H$ ), comptant $34 \%$ d'unités.

99 En vue de la génération de la parole, cette analyse permet de cibler tous les patrons de structures mélodiques, en quatre points primordiaux de leur structure, les cibles externes des mélismes (/ $\mathrm{Ap} /$ et $/ \mathrm{Cp} /$ ), points de jonction entre ligne intonative et mélisme, les cibles internes aux bornes du Noyau (Np/ et Nt/), ainsi que dans leur composition, grâce aux arborescences des familles. Ceci permet de faire bénéficier à la parole synthétique d'une heureuse variabilité évitant la monotonie mélodique trop souvent constatée.

\subsubsection{Harmoniser la mélodie des cibles}

Une étude intéressante a permis de montrer que les cibles mélodiques du mélisme n'étaient pas indépendantes les unes des autres (Caelen-Haumont, 2009).

Le Tableau 6 ci-dessous présentant les taux de corrélation entre les différentes cibles de tous les mélismes, appelle certains commentaires. 
Tableau 6

\begin{tabular}{|c|c|c|c|c|c|c|c|c|}
\hline $\begin{array}{c}\text { Pourcentages de } \\
\text { Corrélations }\end{array}$ & $\begin{array}{c}\text { Np et } \\
\text { Nt }\end{array}$ & $\begin{array}{c}\text { Ap et } \\
\text { Np }\end{array}$ & $\begin{array}{c}\text { Ap et } \\
\text { PT }\end{array}$ & $\begin{array}{c}\text { Ap et } \\
\text { Nt }\end{array}$ & $\begin{array}{c}\text { Cp et } \\
\text { Np }\end{array}$ & $\begin{array}{c}\text { Cp et } \\
\text { PT }\end{array}$ & $\begin{array}{c}\text { Cp et } \\
\text { Nt }\end{array}$ & $\begin{array}{c}\text { Ap et } \\
\text { Cp }\end{array}$ \\
\hline \multirow{4}{400}{ C + DI } & 19 & 74 & -52 & 24 & 12 & 22 & 55 & 14 \\
& 400 & 195 & 195 & 195 & 271 & 271 & 271 & 130 \\
\hline \multirow{2}{*}{329 C } & 19 & 73 & -53 & 21 & 08 & 24 & 53 & 08 \\
& 329 & 172 & 172 & 172 & 238 & 238 & 238 & 120 \\
\hline \multirow{2}{*}{71 D } & 21 & 66 & -43 & 08 & 81 & -44 & 23 & 40 \\
& 71 & 34 & 34 & 34 & 23 & 23 & 23 & 12 \\
\hline 167 C + DI MP & 14 & 63 & 03 & 20 & -03 & 43 & 59 & -04 \\
& 167 & 90 & 90 & 90 & 103 & 103 & 103 & 52 \\
\hline 147 C + DI M & 39 & 78 & -58 & 49 & 34 & -09 & 49 & 35 \\
& 147 & 63 & 63 & 63 & 117 & 117 & 117 & 51 \\
\hline 56 C + DI MF & 25 & 78 & -53 & 40 & 25 & -01 & 44 & 40 \\
& 56 & 34 & 34 & 34 & 45 & 45 & 45 & 28 \\
\hline
\end{tabular}

Taux de corrélations entre les différentes cibles (avec PT, écart entre pied et tête du Noyau) du mélisme (4 locutrices), en fonction des 400 mélismes croissants (C) et décroissants inversés (DI), des 329 croissants, des 71 décroissants, des mélismes croissants et décroissants inversés (167 MP, 147 M, 56 MF). Dans les colonnes, le nombre supérieur indique les taux de corrélation, et le nombre inférieur, l'effectif. Les nombres en gras indiquent les corrélations au-dessus de 0,50 (et symétriquement les anti-corrélations en valeur négative).

102 A savoir tout d'abord comme l'on s'y attendait, que les cibles centrales et extrêmes des mélismes, définitoires, ( $N p$ et $N t)$ ne sont absolument pas corrélées. Cela confirme bien que cet espace de contraste maximal est bien laissé à l'usage personnel et subjectif des locuteurs. Ne sont pas corrélées non plus les valeurs des attaches droite et gauche du mélisme entre elles ( $A p$ et $C p$ ), ou avec les cibles définitoires du mélisme soit respectivement $A p$ avec $N t$, et $C p$ avec $N p$ (sauf pour les mélismes décroissants, ce qui est parfaitement normal car analogue par symétrie à la corrélation $A p$ et $N p$ pour les structures croissantes).

Inversement, pour l'ensemble des 400 mélismes croissants et décroissants inversés, et les croissants seuls, on observe des corrélations satisfaisantes (respectivement 74 et 73) entre les pieds de l'amorce ( $A p)$ et du Noyau ( $N p)$, parfois séparées d'une cible, de même que symétriquement, mais dans des proportions moindres $(55,53)$, entre le pied de la coda $(C p)$ et la tête du noyau (Nt). Les valeurs des taux des types MP (mélismes fin de groupe devant pause), M (internes) et MF (fin de groupe sans pause) montrent comment ils participent aux résultats globaux des 400 mélismes, les M et MF contribuant à l'augmentation des taux entre $A p$ et $N p$, les MP à leur diminution, alors que l'inverse se produit en fin de mélisme pour Nt et $C p$.

$\mathrm{Du}$ fait de la corrélation existant entre $A p$ et $N p$, nous ne sommes pas surpris de constater une corrélation entre le pied de l'amorce $A p$ et l'écart maximal du mélisme $P T$, ou plus exactement une corrélation négative : en effet plus le pied de l'amorce $A p$ (et le pied du noyau $\mathrm{Np}$ ) tend à baisser, et plus l'écart $P T$ tend à augmenter.

Pour rendre les mélismes plus naturels, cette dépendance mélodique entre certaines des cibles des mélismes jamais mise à jour jusqu'à présent, gagne également à être exploitée en synthèse de la parole.

\subsection{Analyse temporelle}

Nous nous intéressons maintenant à l'aspect temporel. La meilleure manière de déterminer un gabarit pour la durée des mélismes est d'utiliser une valeur relative à la 
moyenne temporelle des contextes dans lesquels ils se trouvent et ont été comptabilisés.

\subsubsection{Adopter un gabarit temporel pour les mélismes}

Pour ce faire, nous n'avons pas pris en considération la durée des pauses pour l'établissement de la durée moyenne syllabique des mots dans les groupes.

\subsubsection{Durée des mélismes}

Le Tableau 7 ci-dessous montre la durée relative mélismes / mots en fonction des 4 locutrices, ces dernières étant ordonnées en fonction de l'âge décroissant. Il est intéressant de noter, effet physiologique ou hasard, qu'à la fois la durée moyenne des mots dans les groupes, et celles des mélismes, est dépendante des âges des locutrices, les durées ayant tendance à s'allonger avec l'âge.

Tableau 7

\begin{tabular}{|l|c|c|c|c|c|}
\hline Locutrices & HV1 & SP1 & LR1 & LR2 & Moyenne \\
\hline Durées moyenn es & $259 / 203$ & $247 / 198$ & $214 / 174$ & $189 / 172$ & $909 / 747$ \\
\hline Rapport & 1,28 & 1,25 & 1,23 & 1,10 & 1,22 \\
\hline
\end{tabular}

Durée relative des mélismes en fonction des 4 locutrices par rapport à la durée moyenne syllabique des mots dans les groupes où ils se trouvent.

Bien entendu ces valeurs sont des moyennes, et il est nécessaire de rentrer dans l'analyse des structures pour bénéficier si on le désire, de gabarits temporels mieux adaptés.

110 La Figure 8 ci-dessous s'intéresse à la durée moyenne des mélismes en fonction du nombre de leurs syllabes mélodiques, et en fonction respectivement des 400 mélismes croissants et décroissants, des mélismes croissants, des mélismes décroissants, des 299 mots lexicaux et 101 mots grammaticaux. Bien entendu les populations ne sont pas les mêmes, ce qui explique aussi une part de la variabilité.

111 De manière générale, on constate d'abord que quel que soit le type de mélisme, la durée croît en fonction de leur nombre de syllabes mélodiques, ce qui n'a rien de surprenant.

On peut lire néanmoins sur le graphique que les mélismes lexicaux (Lex, carrés noirs) sont toujours temporellement les plus longs, tandis que les grammaticaux (Gr, carrés gris) sont corrélativement les plus courts. Entre ces deux limites, on trouve deux positions opposées, les mélismes croissants (C, ronds noirs) qui en second lieu sont les plus longs (juste un peu plus courts que les mots lexicaux qui composent l'essentiel des mélismes croissants), et les mélismes décroissants, qui eux sont juste un peu plus longs que les mots grammaticaux. 
Figure 8

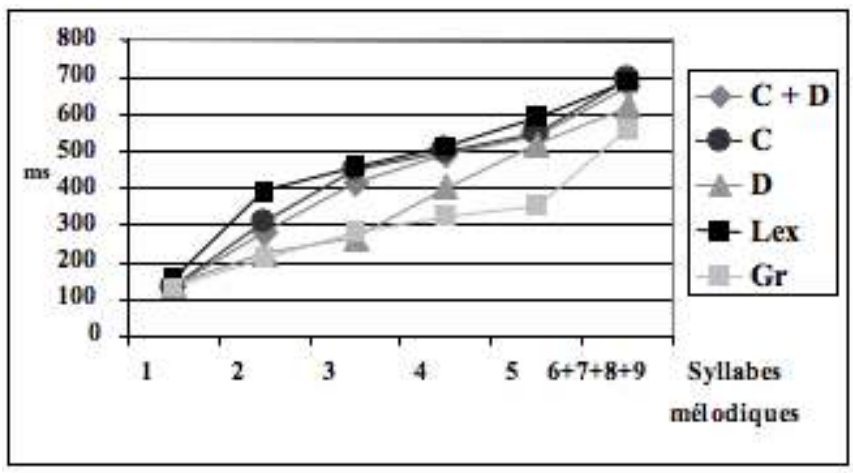

Durée moyenne des mélismes en fonction du nombre de leurs syllabes mélodiques et des catégories de mélismes.

De fait jusqu'à 3 syllabes les courbes des mélismes grammaticaux et décroissants se confondent simplement parce que les mélismes décroissants sont composés en très grande partie de mélismes grammaticaux de 1 et 2 syllabes mélodiques. Au centre de ces différentes courbes, mais plus proche des courbes des mélismes les plus longs du fait que les mélismes croissants sont environ 4,5 fois plus nombreux, on trouve celle qui rassemble mots croissants et décroissants ( $C+D$, losanges gris).

\subsubsection{Durée moyenne des syllabes mélodiques}

114 On peut maintenant affiner les résultats ci-dessus en cherchant à connaître la durée moyenne des syllabes mélodiques en fonction de leur nombre dans le mélisme. C'est l'objet de la Figure 9 ci-dessous.

115 On remarque d'abord un effet inverse au précédent concernant la durée des mélismes : les syllabes mélodiques tendent à être plus courtes quand leur nombre augmente.

Figure 9

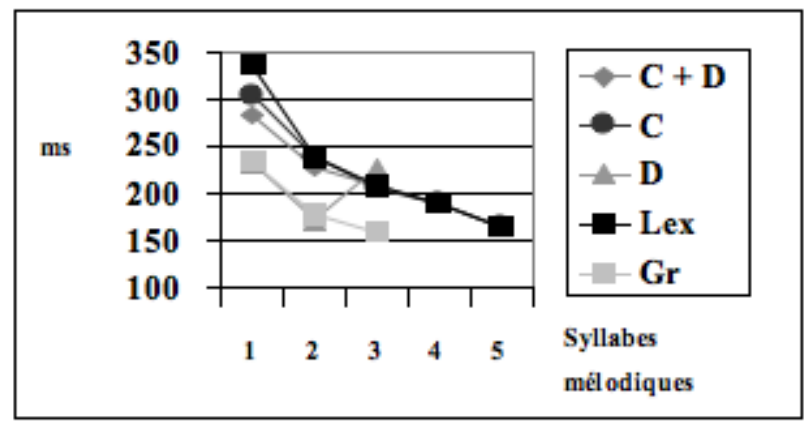

Durée moyenne des syllabes mélodiques en fonction de leur nombre dans le mélisme et des catégories de mélismes.

116 Entre 1 et 2 syllabes mélodiques, la durée se réduit fortement pour les mélismes lexicaux, croissants, croissants joints aux décroissants, et ensuite la diminution est plus progressive. Bien que les mélismes grammaticaux et les mélismes décroissants sont toujours plus courts que les autres, néanmoins leur diminution leur est parallèle sauf pour les décroissants de 3 syllabes, la durée augmentant d'environ $25 \%$. 
117 En fait les choses s'éclairent si l'on considère la nature des monosyllabes mélodiques décroissants : 8 sur 10 sont des mélismes grammaticaux, et les 2 lexicaux sont des monosyllabes composés tous deux de 2 unité phonétiques, tout ceci aboutissant à réduire fortement la moyenne temporelle : il serait donc plus juste de dire inversement que les monosyllabes mélodiques décroissants réduisent leur valeur moyenne de plus de $25 \%$ quand il n'y a pas de lexèmes.

118 Finalement on retrouve le même ordre que précédemment, soit du plus long au plus court : les mélismes lexicaux (1125 syllabes mélodiques évaluées), croissants (1203), croissants et décroissants (1421), les décroissants (218) et enfin les grammaticaux (296), ces deux dernières courbes se confondant pour les mêmes raisons qu'énoncées cidessus.

\subsubsection{Durée des syllabes lexicales}

119 En complément à cette analyse, la durée des syllabes lexicales (et non mélodiques), donc les syllabes des mots lexicaux, fournit un autre ensemble de données qu'il peut être utile de comparer. La Figure 10 ci-dessous montre les courbes moyennes de ces durées, toujours en fonction des mêmes catégories de mélismes. Si le nombre de syllabes lexicales varie de 1 à 5 , les mélismes décroissants et grammaticaux n'ont pas pour leur part un nombre de syllabes supérieur à 2 . De ce fait les valeurs des mélismes croissants et décroissants, croissants et lexicaux sont identiques pour les 4 et 5 syllabes lexicales.

La première constatation, c'est que tout comme la durée des syllabes mélodiques, celle des syllabes lexicales tend aussi à décroître quand le nombre des syllabes mélodiques augmente, même si les valeurs moyennes sont plus élevées pour ce graphique du fait que les mots possèdent moins de syllabes (lexicales) que les mélismes n'ont de syllabes mélodiques. Toutefois on constate que les valeurs des syllabes des mélismes monosyllabiques (figure 9 ci-dessus) sont très différentes en fonction des catégories de mélismes, ce qui est justement l'inverse pour les mots monosyllabiques (Figure $10 \mathrm{ci}$ dessous).

Figure 10

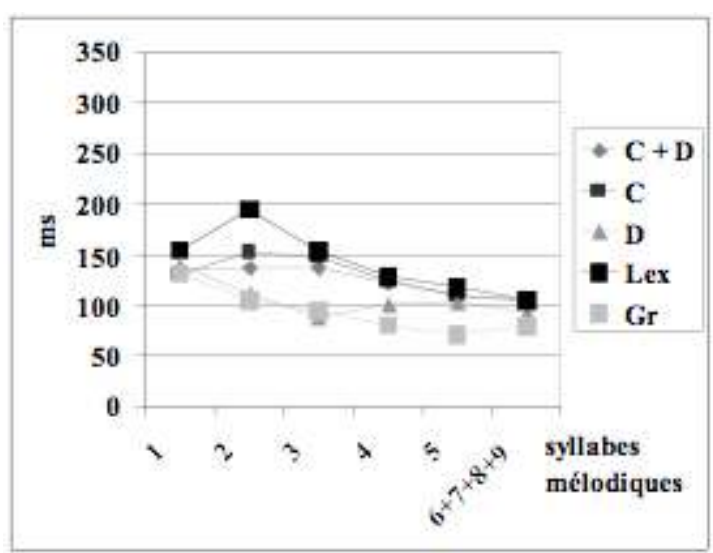

Durée moyenne des syllabes lexicales (et non mélodiques) en fonction du nombre de syllabes mélodiques dans le mélisme et des catégories de mélismes.

121 Les mélismes décroissants de 4 syllabes lexicales (D, triangles gris) semblent à première vue avoir un comportement curieux, avec la remontée de leur durée moyenne. En fait il 
s'agit du même phénomène commenté plus haut : tandis que les mélismes décroissants à 2 syllabes étaient composés essentiellement de mots grammaticaux, les 4 syllabes sont tous inversement des mélismes lexicaux, et de ce fait leur durée moyenne se rapproche des valeurs de leur catégorie.

En conclusion, durée relative des mélismes par rapport aux autres mots, durée moyenne des mélismes, des syllabes mélodiques, des syllabes lexicales en fonction des différents types de mélismes, permettent de donner un gabarit précis pour les mélismes à générer en vue de la synthèse.

\section{Analyse linguistique}

Une autre facette de cette étude en vue de satisfaire les besoins de la génération de parole, concerne l'analyse proprement linguistique des mélismes. Nous aborderons d'abord un point de vue extérieur en replaçant le mélisme dans son contexte linguistique, puis intérieur au niveau des catégories de mélismes. Ces catégories seront envisagées sous plusieurs angles. Mais nous le précisons à nouveau, cette analyse visant à la compréhension des mélismes, reste sur le plan de l'exploitation linguistique (et pragmatique), minimale.

\subsection{Sélectionner les unités lexicales candidates}

Tout d'abord nos données montrent que la localisation du mélisme ne dépend pas de la structuration syntaxique (fin de constituant syntaxique) ou syntagmatique (fin de groupe prosodique). On répertorie en effet des mélismes partout : en finale de groupe prosodique avec ou sans pause, et en position interne. Ainsi parmi nos 400 mélismes, 147 (soit $38 \%$ ) sont des mélismes en position interne (ou M), ce nombre recouvrant d'ailleurs une certaine disparité entre les 4 locutrices (de 23 à $51 \%$ ). Ces $38 \%$ montrent bien que ce phénomène est indépendant de la structuration syntaxique, et qu'il est le propre d'une subjectivité qui cherche à se manifester.

Des études conséquentes pour le français (Caelen-Haumont, 2000, 2004; 2009b...; Morange, 2005; Vuillet, 2005), et pour une langue africaine tonale de la famille bantoue, le bàsàa (Makasso, 2008), ont toutes montré que les mélismes étaient en accord avec l'organisation sémantique et pragmatique du langage, et en particulier avec la structuration isotopique du discours. En effet comme ces travaux l'ont démontré, les champs lexicaux majeurs qui se partagent le texte de l'énoncé fournissent le réservoir des mélismes en fonction des buts explicites ou implicites des locuteurs pour leur discours. Ces champs révèlent les valeurs propres ou de circonstance des locuteurs en fonction du type de communication dans laquelle ils sont engagés. Ils sont généralement construits en arborescence en champs et sous-champs de différents niveaux. C'est donc la voie sémantique et pragmatique ouverte depuis l'origine de nos travaux, qui caractérisera notre méthode.

Pour sélectionner les unités lexicales qui deviendront des mélismes en génération de la parole, on peut procéder pour ce faire à deux moyens différents, un automatique, l'autre manuel. Dans l'attente de procédures informatiques ${ }^{9}$ fonctionnant sur des documents éventuellement de taille restreinte, le recours aux procédures manuelles reste de mise. Deux méthodes complémentaires peuvent être proposées, l'une intuitive, l'autre s'appuyant sur l'organisation formelle des contenus. Ainsi la première voie, à la 
fois rapide et efficace, consiste à parcourir le texte de l'énoncé et à sélectionner intuitivement les mots-clefs.

Une solution qui vise à plus de contrôle, peut envisager par ailleurs de prendre en compte les champs lexicaux, qui regroupent des listes d'unités lexicales partageant la même unité sémantique minimale (ou sème, Rastier, 1985). Dans la mesure où le type de discours éclaire sur les buts et donc finalement sur les thèmes des champs lexicaux, cette méthode demande tout d'abord d'identifier les champs lexicaux majeurs. Ceux-ci seront fonction du type de discours (didactique, scientifique, récit évènementiel, souvenirs, dialogues finalisés, etc.). Il s'agira ensuite de sélectionner certains mots lexicaux, en se fondant sur quelques principes :

- rejeter les termes des sous-champs les plus périphériques (c'est-à-dire les moins prototypiques), ou les moins fournis,

- rejeter les termes les plus périphériques des champs et sous-champs lexicaux majeurs. A titre d'exemple, si dans le champ lexical des fleurs, le terme générique herbe est donné comme synonyme de l'œillet alors herbe ne sera pas choisi comme candidat,

- parmi les champs et sous-champs principaux, sélectionner comme candidat, le mot qui correspond:

- à l'un des mots du titre du document, s'il en existe un,

- au titre des champs et sous-champs, à leurs synonymes, aux concepts-clefs ou aux sèmes majeurs développés par ceux-ci,

- au signifié complexe ou spécialisé (par exemple Cariophyllacées, famille des ceillets),

- au premier item d'un nouveau champ ou sous-champ lexical,

- à un contenu inattendu,

- parmi plusieurs possibilités, au mot le plus spécifique ou précis,

- dans le cas où un même mot est repris plusieurs fois, à sa première occurrence.

Comparant ces deux listes (mots-clefs et mots issus de ces champs lexicaux), on élague et fusionne pour ne retenir qu'une seule liste.

\subsection{Choisir la fréquence des mélismes dans l'énoncé}

l est encore nécessaire par ailleurs de savoir quel est le nombre moyen de mots (exprimé plus précisément en nombre de syllabes lexicales) entre deux mélismes. Bien entendu, il peut varier en fonction des conditions de réalisation, de l'émotion sousjacente du locuteur, du type de discours, du degré de familiarité avec les interlocuteurs ou témoins, ou encore du degré d'appropriation du discours par le locuteur, et en génération de la parole, du degré d'investissement subjectif que l'on veut donner au discours.

Dans le cadre de notre corpus réalisé dans le contexte d'une familiarité moyenne, toutes locutrices confondues, la fréquence entre deux mélismes en fonction des contextes de groupes sélectionnés, est de 6,9 mots. Pour établir cette moyenne, nous avons analysé les 87 fichiers son de notre corpus, chacun totalisant une soixantaine de secondes, soit en tout 5307 secondes (environ 89 minutes), issus des 8 heures de parole. De ces corpus ont été extraits les 400 mélismes avec leur contexte. Parmi ces fichiers ayant donné lieu à une banque de données des mélismes en contexte (cf paragraphe 3.1., Figure 2), nous avons recensé le nombre de syllabes de tous les groupes où figuraient au moins un mélisme. Cette moyenne de 6,875 mots suggère qu'en moyenne, un mélisme est produit tous les 2 groupes de mots comportant de 3 à 4 mots. 
131 Puisque les mélismes sont des unités où s'exprime la subjectivité des locuteurs, il y a tout lieu de considérer que lorsque le nombre de mélismes s'élève dans le discours, la qualité subjective du discours augmente parallèlement.

\subsection{Choisir les différents types de mélismes}

La tâche suivante consiste à choisir les différents types de mélismes possibles. Plusieurs perspectives sont envisagées. Les pages ci-dessous ont recours à des statistiques consistant souvent à recenser le nombre d'items en fonction de différentes catégories. Il est certain que ces résultats dépendent du nombre d'items considérés, en l'occurrence les 400 mélismes étudiés. Une base de données plus importante modifierait peut-être quelque peu les résultats, mais à notre avis, pas fondamentalement car la concordance régulière des résultats entre les locutrices, bien qu'elles appartiennent à 4 générations distinctes, source de grande variabilité potentielle, permet d'avancer l'idée que ces résultats sont consistants.

\subsubsection{Sur le plan contextuel (M, MP, MF, MC)}

Revenons de manière plus explicite sur les mélismes en fonction de leur position dans le groupe : mélisme en fin de groupe devant pause (MP) ou sans pause (MF), en position interne (M), par contact avec un mélisme antérieur ou postérieur (MC).

Concernant les $30 \mathrm{MC}(7.5 \%)$, ils sont tous, à 3 exemples près comme on l'a déjà dit, postérieurs à un mélisme "noyau ", et à deux exceptions près, tous grammaticaux (93\%), la réciproque, comme nous le verrons au paragraphe 5.3.2., n'étant cependant pas vraie, une égale proportion de mélismes grammaticaux pouvant se répartir dans les autres catégories MP, MF et M. Ces MC grammaticaux postérieurs sont au nombre de 25 sur 71 mélismes décroissants, soit $35 \%$. Ils tirent probablement leur origine du fait de la proximité sémantique et syntaxique de l'attracteur qui précède (nom ou verbe) et qu'ils complètent, mais aussi de l'inertie des organes, phénomène qui du reste est lié dans le processus de commande de la production.

Hormis ces $30 \mathrm{MC}$, les autres catégories se composent de 167 mélismes (42\%) devant pause (MP dont 157 croissants, $39 \%$ ), 56 en fin de groupe sans pause (MF $14 \%$, dont 48 croissants soit $12 \%), 147$ internes au groupe (M, 37\%, dont $30 \%$ croissants) et recrutent toutes les classes morphosyntaxiques lexicales. Ces scores sont toutefois dépendants des locuteurs, comme la figure $11^{10} \mathrm{ci}$-dessous le montre.

Figure11

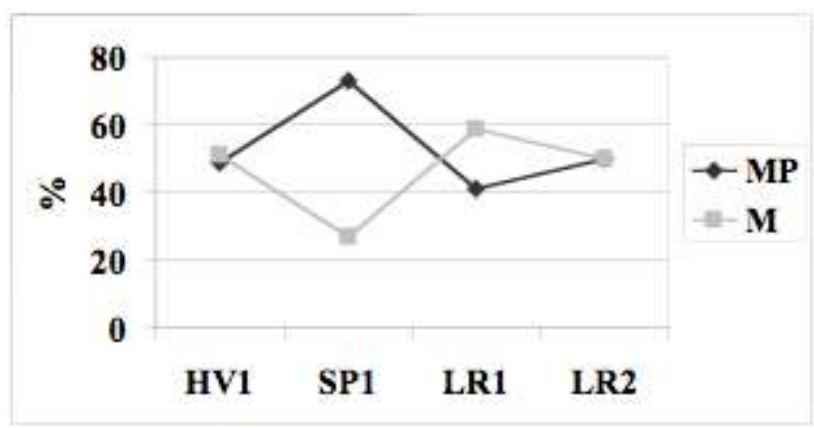

Distribution en pourcentages des mélismes devant pause (MP) et internes au groupe (M) en fonction des 4 locutrices et de l'ensemble des mélismes MP + M. 
Les scores ayant plutôt tendance à s'équilibrer, la distribution finale montrant une majorité pour les MP est due à une forte proportion de ceux-ci chez la locutrice SP1. Pour cette dernière, la fonction des mélismes serait plus souvent liée aux frontières de groupe que chez les autres. S'agit-il d'une fonction syntagmatique, voire syntaxique ? De toutes les façons fonctions syntaxiques, expressives ou subjectives peuvent se superposer.

\subsubsection{Sur le plan morphosyntaxique $(L, G)$}

Si toutes les classes morphosyntaxiques sont recrutées par les différentes catégories de mélismes, toutefois parmi les 299 lexèmes mélismés, croissants et décroissants, les noms sont les plus nombreux (respectivement pour les mélismes croissants et décroissants, $58 \%$, (dont MP, $31 \%, \mathrm{M}, 19 \%, \mathrm{MF}, 8 \%$, tandis que MC significativement ne recrute aucun nom), et loin devant les autres classes morphosyntaxiques (Adjectifs + participes passés : $16 \%$, Verbes, $12 \%$, Noms propres, $9 \%$, Adverbes, $5 \%$, Pronoms indéfinis, $1 \%$ ). Dans l'ensemble, au sein de ces 299 mélismes, MP, M, et MF se distribuent respectivement en 48,35 et $17 \%$.

Nous avons déjà précisé au paragraphe 5.3.1. que les $\mathrm{MC}$ sont tous, à deux exceptions près, des mots grammaticaux (ou $\mathrm{G}$ ). Toutefois la réciproque montre que certains mots grammaticaux appartiennent aussi aux mélismes croissants mais dans une proportion plus faible ( 55 sur 329 , soit $17 \%$ ), et parmi ces 55 , les types se répartissent assez équitablement entre les mélismes en fin de groupe (MP, $40 \%$, MF, $7 \%$, soit en tout $47 \%),-$ dont l'intonation le plus souvent ascendante en cette position explique la forme croissante de ces mots grammaticaux-, et internes au groupe (M, $47 \%$ ), les MC par ailleurs étant rares avant un autre mélisme (MC, $5 \%$ ).

Si l'on comptabilise les mots grammaticaux croissants et les nombreux décroissants, les proportions se modifient (MP $23 \%$, MF $6 \%$, soit $29 \%$ en tout; M $42 \%$; MC $30 \%$ ), qui bénéficient de l'apport des décroissants pour le type $M$, faisant de cette dernière, la catégorie la plus nombreuse.

Bien que les mélismes décroissants sont prioritairement composés de mots grammaticaux, il n'en reste pas moins vrai que dans le cadre des 400 mélismes, les grammaticaux croissants sont statistiquement légèrement plus nombreux que les décroissants (14\% contre $12 \%$ ), ou que les 101 mots grammaticaux ( $54 \%$ contre $46 \%$ ).

Concernant les 299 lexèmes (L), ils se répartissent en 274 mélismes croissants $(91 \%)$ et 25 décroissants (9\%), soit dix fois moins. Le Tableau 8 ci-dessus présente les effectifs (en pourcentages) de ces 299 lexèmes. Les pourcentages sont calculés (axe vertical) dans le cadre de chaque type de mélisme (MP, MF, $M$ et $M C$ ). Ces effectifs étant très proches (identiques ou à 1 ou $2 \%$ près selon le cas) de ceux des mélismes croissants, nous ne les présenterons donc pas. En premier lieu ce sont les noms qui sont mélismés quelle que soit leur position en finale de groupe (avec ou sans pause), ou en position interne. 
Tableau 8

\begin{tabular}{|c|c|c|c|c|c|}
\hline Mélismes Croissants et Décroissants & MP & MF & M & MC & Total \\
\hline Noms communs & 65 & 47 & 53 & 0 & 58 \\
\hline Noms propres & 7 & 12 & 10 & 0 & 9 \\
\hline Verbes & 9 & 12 & 15 & $(1 \mathrm{occ})$ & 10 \\
\hline Adjectifs et participes passés & 14 & 24 & 16 & 0 & 16 \\
\hline Adverbes & 6 & 4 & 6 & 0 & 5 \\
\hline Pronoms indéfinis & 0 & 2 & 1 & 0 & 1 \\
\hline Total & 100 & 101 & 100 & $(1 \mathrm{occ})$ & 99 \\
\hline
\end{tabular}

Pourcentages des 299 mélismes lexicaux croissants et décroissants selon les différentes catégories morphosyntaxiques en fonction des types de Mélismes (MP devant pause, MF en fin de groupe sans pause, $\mathrm{M}$ en position interne de groupe, $\mathrm{MC}$ par contact).

Les figures 12 (101 mots grammaticaux) et 13 (299 mots lexicaux) ci-dessous présentent la répartition des mélismes dans les 4 types de mélismes (MP, MF, M, MC) en fonction du nombre de leurs syllabes mélodiques.

143 On constate tout d'abord sans surprise que le nombre des mélismes grammaticaux comporte généralement moins de syllabes (de 1 à 3), alors que les lexicaux en comportent le plus souvent de 3 à 4 . Avec l'ensemble des mélismes grammaticaux et lexicaux, ce sont les 4 syllabes mélodiques qui dominent (147 unités) puis les 3 syllabes (135).

144 Par ailleurs parmi les grammaticaux, tous les types sont présents, ce qui n'est pas le cas pour les lexicaux malgré leur nombre trois fois plus élevé : alors que les MC sont bien représentés dans les mots grammaticaux, ils sont en effet quasi inexistants parmi les lexicaux. Ceci montre très clairement que le mélisme est un processus essentiellement monolexical, au contraire des émotions, comme on l'a vu dans l'introduction, qui affectent des groupes ou une suite de groupes.

\section{Figure 12}

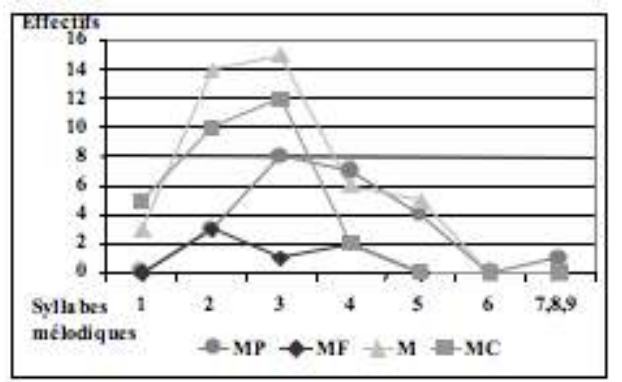

Mots grammaticaux : répartition des 101 mélismes dans les 4 types de mélismes (MP, MF, M, MC) en fonction du nombre de leurs syllabes mélodiques 
Figure 13

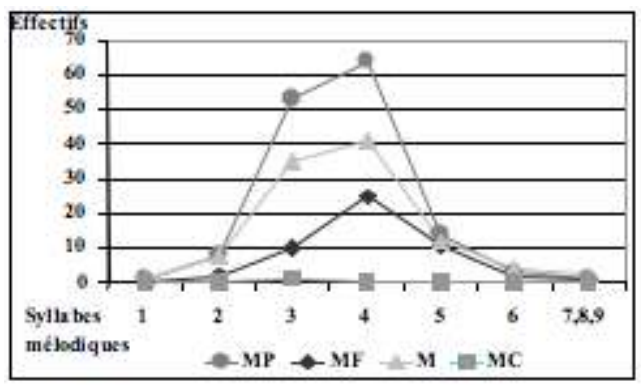

Mots lexicaux : répartition des 299 mélismes dans les 4 types de mélismes (MP, MF, M, MC) en fonction du nombre de leurs syllabes mélodique

Par ailleurs les MP étant une catégorie assez peu représentée parmi les grammaticaux, ils représentent inversement la catégorie la plus nombreuse pour les lexicaux (comme on l'a vu, sous l'effet d'un grand nombre de ceux-ci chez la locutrice SP1). Chez les grammaticaux, c'est inversement le type $\mathrm{M}$ qui est le plus fourni, avant le type MC. Toutes ces remarques correspondent bien aux comportements attendus des mots grammaticaux et des lexèmes, les premiers étant souvent antéposés aux seconds.

\subsubsection{Sur le plan des fonctions grammaticales}

Une étude complémentaire concerne les fonctions des noms communs (Tableau 9 cidessous). Au nombre de 172, les noms communs représentent $43 \%$ des 400 mélismes (ou $58 \%$ des mélismes lexicaux). Selon les locutrices, ils totalisent 35 à 57 unités.

147 La première constatation, c'est une assez grande homogénéité des résultats, quels que soient l'âge des locutrices, et les sujets des discours. Trois catégories regroupent la majorité des mélismes, à savoir par ordre décroissant, les compléments circonstanciels (en gras, $38 \%$ en moyenne), les COD (en italiques, $35 \%$ ) et les compléments prépositionnels (soulignés, $20 \%$ ). Par " complément prépositionnel », nous entendons les compléments de noms, d'adjectifs, de participes passés et d'adverbes. Si nous regroupons tous les compléments introduits indirectement et avec eux les circonstanciels directs, cette catégorie regroupe $59 \%$ des noms.

Tableau 9

\begin{tabular}{|c|c|c|c|c|c|c|c|}
\hline $\begin{array}{c}\text { Fonction } \\
\text { des noms }\end{array}$ & S & COD & Att & App & COI & $\begin{array}{c}\text { Complément } \\
\text { prépositionnel }\end{array}$ & $\begin{array}{c}\text { Complément } \\
\text { circonstanciel }\end{array}$ \\
\hline HV1 & 4 & 38 & 0 & 2 & 0 & 10 & 46 \\
\hline SP1 & 4 & 33 & 2 & 0 & 2 & 25 & 35 \\
\hline LR1 & 8 & 34 & 0 & 0 & 0 & 29 & 29 \\
\hline LR2 & 3 & 31 & 3 & 3 & 0 & 20 & 40 \\
\hline Total & 4 & 35 & 1 & 1 & 1 & 20 & 38 \\
\hline
\end{tabular}

Pourcentages des différentes fonctions ( $\mathrm{S}$ : sujet, COD : complément d'objet direct, Att : attribut, App : apposition, $\mathrm{COI}$ : complément d'objet indirect), des noms propres en fonction des 4 locutrices (par ordre décroissant d'âge : HV1, SP1, LR1, LR2).

Une autre constatation, c'est le nombre extrêmement faible de la fonction sujet, 4 \% en moyenne, dû peut-être à l'emploi substitutif des pronoms personnels, mais aussi sans doute à leur position en tête de groupe. Les autres catégories sont pratiquement inexistantes. 
149 Nous retenons donc que les types de compléments (circonstanciels, prépositionnels et COD) fournissent la très grande partie des mélismes nominaux ( $93 \%$ d'entre eux), la catégorie des noms correspondant à elle seule $43 \%$ des 400 mélismes. Ces résultats sont concordants entre les 4 locutrices et ce, dans un contexte de langage spontané.

\section{Démonstration expérimentale}

Une fois que les mots candidats pour devenir des mélismes ont été sélectionnés (cf paragraphe 5.1. ci-dessus), il faut apparier les valeurs intonatives aux bornes du mot candidat avec les valeurs propres au mélisme. Pour ce faire on a recours au dictionnaire des familles mélodiques (cf paragraphe 4.1.6.3. ci-dessus, et annexe paragraphe 3.4.). Ce dernier recensant toutes les structures attestées, propose différents patrons classés en fonction des cibles mélodiques initiale et finale, comme on le sait, points d'ancrage du mélisme sur la courbe d'intonation. Il suffit alors au moteur de recherche de la base de données, d'extraire les patrons qui présentent les mêmes cibles initiale et finale que les mots à mélismer et de doter ce mot candidat d'un patron correspondant.

En annexe, on trouvera expliquée en détail et illustrée de recopies d'écran, la méthode présentée ici, et décrivant les 8 exemples de mélisme lexicaux et grammaticaux qui ont été créés, à pente mélodique descendante, ascendante ou en plateau. Les exemples comportent $\left(1^{\circ}\right.$ les originaux portant les numéros impairs, $2^{\circ}$ les phrases " resynthétisées » avec l'adjonction de mélismes portant les numéros pairs,) soit 1 seul mot (ex n 1-2, 3-4, 5-6), 2 mots (ex n 7-8), ou 3 mots (ex n 9-10) modifié(s) dans chaque énoncé, phrase entière ou portion.

A titre de première illustration, nous avons appliqué manuellement cette procédure à des extraits de parole naturelle par le moyen de l'analyse/synthèse proposée par Praat. Dans le corpus, parmi d'autres exemples disponibles bien entendu, nous avons donné la préférence à un énoncé dont la prosodie se montrait en rupture avec la sémantique du fait de la nostalgie de la locutrice. En effet la locutrice SP1 prononce l'énoncéj'étais toujours partante pour ce genre de distraction sur le mode du regret d'une époque lointaine et de la tristesse. Cet exemple est donc idéal pour transformer cet « anti-mélisme » en mélisme, toutes choses égales par ailleurs (intensité, durée).

Pour ce faire afin de supprimer les dissonances entre intonation et mélisme, nous avons cherché dans le dictionnaire des familles de mélismes, un exemplaire ayant les mêmes cibles mélodiques initiale du mélisme (/Ap, ayant comme valeur ici le $2^{\mathrm{e}}$ niveau dans les graves $/ \mathrm{i} /)$ et finale $\left(/ \mathrm{cp} /\right.$, ayant le niveau d'une valeur moyenne, soit $4^{\mathrm{e}}$ niveau ou $/ \mathrm{c} /$ ). Seul le patron mélodique de partante est modifié. On peut donc évaluer l'apport strict du changement de structures mélodiques dû par le seul effet du mélisme.

154 Ainsi 2 patrons mélodiques (mélodiquement très proches l'un de l'autre d'ailleurs) ont été trouvés, prononcés par une autre locutrice (LR1). Le Tableau 10 ci-dessus présente les structures mélodiques des 3 mots, le non-mélisme partante locutrice SP1), et les mélismes vigne et monde (locutrice LR1). Ce sont des structures du niveau 4 de profondeur (/ib bH Hh hc/ ou /ii iH Hh hc/) -ce qui équivaut aussi au nombre de syllabes mélodiques- qui peuvent être substituées à la structure non mélismée partante de niveau 3 de profondeur (/ic $c b b c /$ ). 
Tableau 10

\begin{tabular}{|c|c|c|c|c|c|c|c|c|}
\hline \multirow[t]{2}{*}{ Source } & \multirow{2}{*}{$\begin{array}{l}\mathrm{N}^{\circ} \mathrm{du} \\
\text { mélisme }\end{array}$} & \multirow{2}{*}{$\begin{array}{l}\text { Type de } \\
\text { mélismes }\end{array}$} & \multirow[t]{2}{*}{ Locutrice } & \multirow[t]{2}{*}{ Lexique } & \multicolumn{4}{|c|}{$\begin{array}{l}\text { Niveaux de profondeur / nombre } \\
\text { de syllabes mélodiques }\end{array}$} \\
\hline & & & & & 1 & 2 & 3 & 4 \\
\hline $\begin{array}{c}\text { Enoncé : } \\
\text { Non-mélisme }\end{array}$ & l & l & SP1 & partante & ic & ic $c$ & ic $c b b c$ & 1 \\
\hline \multirow{2}{*}{$\begin{array}{l}\text { BD : } \\
\text { mélismes }\end{array}$} & 228 & M & LR1 & vigne & ic & $\mathrm{iH} \mathrm{Hc}$ & $\mathrm{iH} \mathrm{Hh}$ he & $\mathrm{ib} \mathrm{bH} \mathrm{Hh} \mathrm{hc}$ \\
\hline & 263 & $M$ & LR1 & monde & ic & $\mathrm{iH} \mathrm{Hc}$ & $\mathrm{iH} \mathrm{Hh} \mathrm{he}$ & ii iH Hh he \\
\hline
\end{tabular}

Patrons mélodiques des mélismes («vigne », « monde », locutrice LR1) ayant les mêmes cibles initiale et finale que le mot candidat « partante » (locutrice SP1) à mélismer. Les niveaux 2 et 3 indiquent la filiation depuis la racine (ou famille) /ic/.

La substitution s'effectue dans le fichier Manipulations de Praat.

Figure 14

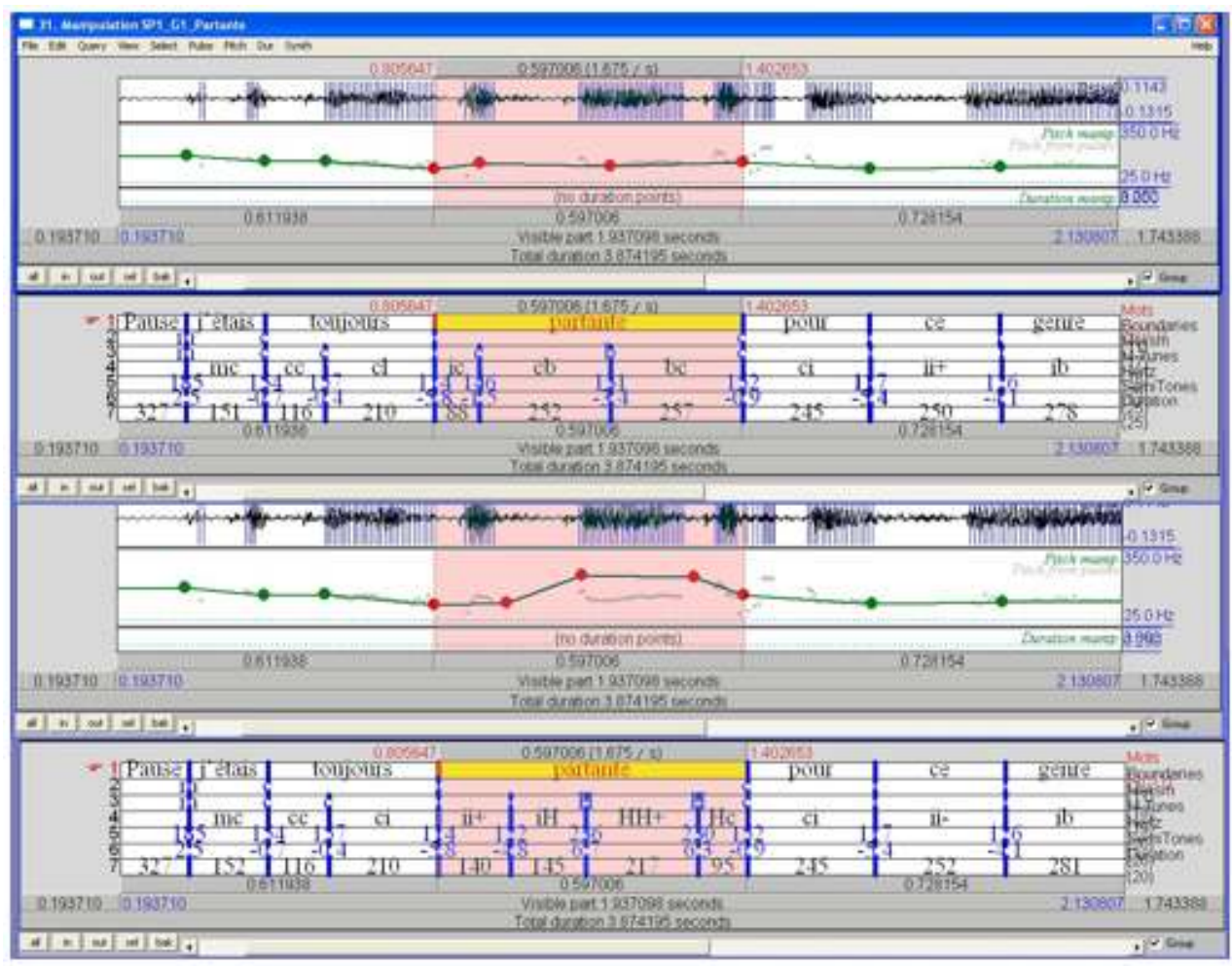

En haut, fenêtre 4, structure mélodique originale du mot candidat « partante » (locutrice SP1) non mélismé dans son énoncé (cf la courbe mélodique plate et plutôt grave, dessous fenêtre 3 , ses valeurs mélodiques correspondantes (valeurs des syllabes mélodiques /ic cb bc/) dans le TextGrid, calculées automatiquement avec leurs valeurs en $\mathrm{Hz}$, demi-tons et leur transcodage alphabétique) puis en comparaison, fenêtre 2, la courbe mélodique de l'énoncé modifié, et dessous, fenêtre 1, le TextGrid avec les nouvelles valeurs mélodiques (/ii iH HH Hc/).

La figure 14 ci-dessus confronte mélodie originale et mélodie modifiée, les modifications des structures mélodiques apportées au mot partante le transformant en mélisme.

On observe ainsi par comparaison sur cette figure que le mot partante, possédant un contour assez plat dans les tons moyens et graves (de 2 à 4 sur une échelle de 9 Lien vers exemple sonore $n^{\circ} 1$ ), n'a rien de comparable avec une structure de mélisme qui 
compte au moins un niveau haut ou aigu $H, S$ ou $A$ (soit de 7 à 9 Lien vers exemple sonore 2).

Les exemples attestés dans notre banque de données, permettent de lui attribuer un niveau maximal en $\mathrm{H}$ (niveau 7 sur 9). Il est probable que d'autres structures plus aiguës en $S$ (niveau 8) ou A (niveau 9) sont également possibles. Mais du fait du registre mélodique relativement bas de l'énoncé, le niveau $\mathrm{H}$, comme on l'a précisé au paragraphe 4.1.1. ci-dessus, est suffisant pour qu'une proéminence auditive soit clairement perçue ( $\mathrm{cf}$ l'exemple sonore original et modifié $\mathrm{n}^{\circ} 1$ et 2 ).

On note aussi que la fin de la phrase naturelle est prononcée avec une intensité beaucoup plus forte. Ceci est dû au fait que la locutrice cherchait ses mots (les hésitations ont été coupées pour rendre l'énoncé plus compact), et lorsqu'elle a trouvé ce mot, elle l'a doté d'une nouvelle structure de mélisme et d'une énergie forte. Rien n'a été modifié à ce propos.

Les échantillons de parole originaux et modifiés sont fournis avec cette communication, commentés et illustrés en annexe, et peuvent donc être évalués auditivement. Comme on peut en juger, la modification, toutes choses égales par ailleurs, est perçue de manière très significative, et avec un effet totalement naturel.

\section{Conclusion}

Cette étude générale est fondée de manière originale sur une analyse fine et exhaustive des structures mélodiques composant les proéminences prosodiques subjectives ou mélismes, ce qui n'a jamais été entrepris jusqu'à ce jour dans le domaine de la génération de la parole (ni même en analyse prosodique).

Deux grandes parties permettent de décrire le processus des mélismes sous l'angle tout d'abord prosodique, puis sous l'angle linguistique en limitant ce dernier à ce dont les spécialistes de génération ont besoin.

Des informations sont tout d'abord données au niveau prosodique concernant la définition des mélismes, leur forme globale (pentes Croissante, Décroissante, Plateau), leur composition interne en formes de cibles et de syllabes mélodiques, (cibles et syllabes mélodiques de l'amorce, noyau et coda, et sous-parties) avec indication des fonctions de ces cibles, de leur présence obligatoire ou facultative, des statistiques, des motifs principaux des syllabes mélodiques, de patron prototypique.

Des informations originales sont également fournies pour créer et étoffer les contrastes internes des mélismes grâce à l'étude de deux procédés (symétrie et duplication) recensés dans notre corpus, sur leur usage en combinaison en fonction des 4 locutrices, sur les structures arborescentes des mélismes et des informations quantitatives relatives aux « familles » mélodiques en particulier constitutives du Noyau.

Des résultats quantitatifs nouveaux ont par ailleurs mis à jour un processus d'harmonisation et de corrélation mélodique entre certaines cibles des mélismes.

Toujours dans le domaine prosodique, le volet de la durée a permis aussi de fournir des gabarits temporels des mélismes relativement d'une part aux mots composant les groupes prosodiques, et d'autre part en fonction du nombre de leurs syllabes mélodiques, ainsi que des gabarits des syllabes mélodiques, des syllabes lexicales, les 
deux en fonction de leur nombre dans les mélismes. Bien sûr des informations encore plus précises sont disponibles en fonction des locutrices.

Sur le plan linguistique, l'analyse précise tout d'abord la méthode pour détecter les mots d'un texte susceptibles de devenir des mélismes au niveau prosodique. Par ailleurs des précisions sont apportées sur la fréquence des mélismes, sur leurs caractéristiques en fonction des types selon leur position dans le groupe (MP en finale devant pause, MF en finale sans pause, $\mathrm{M}$ interne au groupe, $\mathrm{MC}$ par contact), sur les fréquences de ces types en fonction des catégories morphosyntaxiques (noms communs, propres, adjectifs, participes passés, verbes, adverbes, pronoms indéfinis et mots grammaticaux), des fonctions du nom (sujet, complément d'objet direct et indirect, attribut, apposition, complément prépositionnel, circonstanciel).

Ensuite des informations détaillées sont données pour structurer à un autre niveau la " chair » des mélismes, en termes de fréquence de mélismes en fonction de l'opposition mots lexicaux/grammaticaux et en fonction des positions dans le groupe (MP, M, MF, $\mathrm{MC})$.

$\mathrm{Vu}$ la généralité de cette étude qui repose sur des considérations mélodiques et prosodiques, il y a lieu de penser que la méthode est applicable à d'autres langues, que celles-ci soient accentuelles, tonales ou autres, et même aux chants et cris des espèces animales, permettant de relever et d'annoter des structures types en fonction de leurs communications, des comportements sociaux, des contraintes biologiques, des alertes...

Nous espérons que cette étude exhaustive grâce à toutes les informations fournies, est susceptible non seulement de renouveler les méthodes, mais aussi de fournir une aide substantielle pour la génération de la parole permettant de composer les mélismes à l'identique du nature, ou à tout le moins de s'en approcher.

\section{BIBLIOGRAPHIE}

Acapela Group, http://www.acapela-group.com.AT\&T Natural Voices, http://

www.naturalvoices.att.com/Audibert, N. (2008) : Prosodie de la parole expressive : dimensionnalité d'énoncés méthodologiquement contrôlés authentiques et actés, Thèse de doctorat de Grenoble INP, spécialité Ingénierie de la Cognition, de la Création et des Apprentissages.

Boersma, P. Weenink, D., (2012): Praat: doing phonetics by computer ", http://www.praat.org/).

Boula de Mareüil, P., Célérier, P., Cesses, T., Fabre, S., Jobin, C., Le Meur, P.-Y., Obadia, D., Soulage, B., Toen, J. (2001) : Elan Text-To-Speech : un système multilingue de synthèse de la parole à partir du texte. Traitement Automatique des Langues 42(1), 223-252.

Boula de Mareüil, P., D’Alessandro, C., Raake, A., Bailly, G., Garcia, M.-N., Morel, M. (2006): A joint intelligibility evaluation of French Text-To-Speech systems: the EvaSy SUS/ACR campaign. Proceedings of the 5th international Conference on Language Resources and Evaluation (LREC 2006), Genoa, Italy, 2034-2037. 
Black, P., Taylor, P. (1997) Assigning phrase breaks from part-of-speech sequences. Proceedings of Eurospeech Conference, Rhodes, 995-998.

Caelen-Haumont, G. (1978) : Structures prosodiques de la phrase énonciative simple et étendue. Thèse de Doctorat de 3ème cycle en Sciences du Langage : Université du Mirail, Toulouse.

Caelen-Haumont, G. (1981), Hamburg Buske: Hamburger Phonetische Beitrage, Band 34. ISBN

3-87118-463-463.

Caelen-Haumont, G. (1991) : Stratégies des locuteurs en réponse à des consignes de lecture d'un texte : analyse des interactions entre modèles syntaxiques, sémantiques, pragmatique et paramètres prosodiques. Thèse de doctorat d'état : Université d'Aix-en-Provence, 577 p.

Caelen-Haumont, G. (2002) : Prosodie et dialogue spontané : valeurs et fonctions perlocutoires du mélisme. Travaux Interdisciplinaires du Laboratoire Parole et Langage d'Aix (TIPA), no. 21.,13-24. ISSN 1621-0360.

Caelen-Haumont, G. (2004) : Valeurs pragmatiques de la proéminence prosodique lexicale : de l'outil vers l'analyse. Actes des 25 èmes Journées d'Etude sur la Parole JEP, 19-22 avril 2004, Fès, Maroc, 105-108. [hal-00241546 - version 1]

Caelen-Haumont, G. (2009a) : Prosodie et sens : une approche expérimentale, Volume 1 (216 p.) et 2 (262 p.). Paris, l'Harmattan-Marges Linguistiques, ISBN 978-2-296-06625-0 et ISBN : 978-2-296-06626-7.

Caelen-Haumont, G. (2009b): Emotion, emotions and prosodic structure: an analysis of the melisms patterns and statistical results in the spontaneous discourse of 4 female speakers from four generations. Chapitre d'ouvrage, in Sylvie Hancil éd., The Role of Prosody in Affective Speech, Peter Lang, li97, 95-138.

Caelen-Haumont, G., (2010): F0 prominences (melisms): a deeper insight about morphophonology, Speech Prosody 2010 100048:1-4, in ed. Mark Hasegawa-Johnson, University of Illinois at Urbana-Champaign, http://www.speechprosody2010. illinois.edu/papers/100048.pdf, Chicago, USA.

Caelen-Haumont, G., Bel, B. (2000) : Le caractère spontané dans la parole et le chant improvisés : de la structure intonative au mélisme. Revue PArole, $\mathrm{n}^{\circ}$ 15-16, p. 251-302. [hal-00256388 - version 1]

Caelen-Haumont, G., Auran, C. (2004): The phonology of Melodic prominence: the structure of melisms. Actes de Speech Prosody SP2004, Nara, Japon, 143-146. [hal-00256390 - version 1]

Coorman, G., Fackrell, J., Rutten, P., Van Coile, B. (2000): Segment selection in the L\&H RealSpeak Laboratory TTS system. Proceedings of ICSLP, Beijing, 395-398.

D’Alessandro, C., Boula de Mareüil, P., Garcia, M.-N., Bailly, G., Morel, M., Raake, A., Béchet, F., Véronis, J. Prudon, R. (2008) : EVASY, évaluation des synthétiseurs de parole en français in S. Chaudiron \& K. Choukri (dir.), L'évaluation des technologies de traitement de la langue : les campagnes Technolangue. Paris, Lavoisier, 183-208.

Durand, J., Laks, B., Lyche, C. (2005) : Un corpus numérisé pour la phonologie du français. In G. Williams (ed.) La linguistique de corpus. Rennes, Presses Universitaires de Rennes, 205-217.

Dutoit, T., Pagel, V., Pierret, N., Bataille, F., Van der Vrecken, O. (1996): The MBROLA project: towards a set of high quality speech synthesizers free of use for non commercial purposes. Proceedings of ICSLP, Philadelphia,1393-1396. 
FESTIVAL, Centre for Speech Technology Research), Université d'Edinburgh, Angleterre, http:// www.cstr.ed.ac.uk/ projects/festival/CSTR.

Dutoit, T. et Leich, H. (1993): An analysis of the performances of the MBE model when used in the context of a Text-To-Speech system. Actes d'Eurospeech 93, Berlin, 531-534.

Ehrmann, M. (2008) : Les Entités Nommées, de la linguistique au TAL : statut théorique et méthodes de désambiguïsation, Thèse de doctorat, Université Paris 7 Diderot.

Hernandez, N., (2004) : Description et détection automatique de structures de texte, Doctorat de l'Université d'Orsay Paris XI, 1-285.

Hirst D., (2007): A Praat plugin for Momel and Intsint with improved Algorithms for modelling and coding intonation, Proceedings of ICPhS XVI, Saarbrucken, Germany, 1233-6.

Le Beux, S., Rilliard, A. \& d'Alessandro, C., Calliphony (2007): A real-time intonation controller for expressive speech synthesis. Proceedings of the 6th ISCA Speech Synthesis Research Workshop, Bonn, Germany.

Ljungqvist, M., Lindstrom, A., Gustafson, K. (1994): A new system for text-to-speech conversion, and its application to Swedish. Actes de l'International Conference of Spoken language Processing ICSLP, 1779-1782.

Mac Dang Khoa (2012) : Génération de parole expressive dans le cas des langues à tons, thèse de doctorat Signal, Image, Parole, Télécoms, Université de Grenoble.

Makasso, E-M. (2008) : Intonation et mélismes dans le discours oral spontané en bàsàa, thèse de doctorat en sciences du langage. Université de Provence Aix-Marseille.

Moore, J. D. and Pollack, M. E. (1992): A problem for RST: The need for multi-level discourse analysis. Computational Linguistics, $18(2)$ : 537-544.

http://www.cs.pitt.edu/ jmoore/papers/cl-squib.ps.

Morange, S. (2005) : Approches structurale, prosodique, psycho-cognitive de quelques propriétés linguistiques subjectives de la parole : l'exemple de trois Montmartrois âgés. Thèse de doctorat en sciences du langage, Université de Paris 3.

Orange Labs, http://www.orange.com/fr_FR/innovation/inventer/index.html

Phonologie du Français Contemporain, Durand, J., Laks, B., Lyche, C., http://www.projet-pfc.net/

Poibeau, T. (2008.) : Des mots au texte: Analyse sémantique pour l'accès à l'information. Mémoire HDR, Laboratoire d'Informatique Paris-Nord, 1-130.

Rastier, F. (1985) : L'isotopie sémantique, du mot au texte. Thèse de Doctorat d'Etat, Université de Paris IV.

Scansoft / Nuance, http://www.nuance.fr

Ségalen, L. et Cadic, D. (2008) : Introduction d'éléments paralinguistiques en synthèse vocale. Actes des 27 JEP, Avignon.

Suciu, I., Kanellos, I., Moudenc, T. (2007) : Expressivité et synthèse vocale. Isotopies expressives, cohérence discursive et structures prosodiques. Nouveaux cahiers de linguistique française, 28 (2007), 199-206.

Vuillet, J. (2005) : La part de l'expressivité vocale dans le réglage de l'interaction et dans l'émergence de conduites langagières lors d'un atelier de langage en début d'école maternelle. Thèse de doctorat en sciences de la communication, Université Stendhal-Grenoble III. 
Weber B., Caelen-Haumont G., Pham B. H., Tran D-D, (2012): MISTRAL: A Melody Intonation Speaker Tonal Range semi-automatic Analysis using variable Levels, Proceedings of LREC 2012 http://www.lrec-conf.org/proceedings/lrec2012/index.html.

Whistler, Microsoft, http://research.microsoft.com/research/srg/ssproject.aspx.

\section{ANNEXES}

\section{Méthode de génération des formes mélodiques de mélisme en « resynthèse » vocale}

Dans les noms des fichiers sonores soumis à l'écoute, les nombres impairs correspondent à la version naturelle et spontanée, non modifiée, inversement les nombres pairs, à la version mélismée. Dans les noms des fichiers sonores :

- P signifie Plateau mélodique (même niveau au sein des 9 possibles)

- M, pente Montante

- D, pente Descendante.

Pour illustrer la présente communication, 8 mots ont été « resynthétisés » dans 5 phrases différentes. Ce terme de « resynthèse » est utilisé dans la communauté des technologies de la parole pour exprimer que la synthèse est produite à partir de la phrase naturelle et non par exemple de tout petits échantillons de parole préalablement stockés ${ }^{11}$.

Nous avons ainsi emprunté 5 phrases ou propositions à 4 locutrices sur les enregistrements desquels une large étude antérieure a porté (Caelen-Haumont, 2009b ; 2010). Cette étude une fois achevée sur 400 mélismes (100 mélismes $x 4$ locutrices), nous avons ensuite réalisé sous excel un dictionnaire de mélismes, comportant toutes les références de ces derniers, classées en 2 parties : mélismes de mélodie croissante (y compris les plateaux très peu nombreux) et mélismes de mélodie décroissante.

Pour offrir un panorama un peu complet, nous avons diversifié nos exemples tant sur le plan de la morphosyntaxe, de leur position par rapport au groupe, que de la forme des pentes (montantes, descendantes, en plateau).

\section{Mots lexicaux et grammaticaux}

Pour les exemples de resynthèse que nous proposons, nous avons choisi à la fois : des phrases dont les unités (mot commun ou propre, mot grammatical) et/ou la signification globale de l'énoncé offraient un sens susceptible de convenir à une mise en valeur mélodique (c'est-à-dire à un mélisme) : mots lexicaux évoquant une dynamique psychologique (/partante/), un lien affectif (/grand-mère/, /Soulac/), un motclef du champ lexical (/poissons/), un fréquentatif (/souvent/), mots grammaticaux tels qu'un adverbe d'intensité redoublé (/très très/), et le pronom personnel de première personne (/moi/), qui plus est, en position focale, et dont ces mots précisément n'étaient précisément pas des mélismes. Bien au contraire, ces phrases ou extraits de phrases sont prononcés sur un registre assez plat, ressemblant souvent à des énoncés 
de synthèse. C'est la raison pour laquelle nous avons choisi ces différents extraits de parole spontanée, en leur laissant toutes leurs caractéristiques, sauf la mélodie dans le mot concerné.

\section{Mélismes montants, descendants, en plateau}

De la même façon, nous avons choisi des mélismes dont initialement les mots mélodiquement repérés par leurs valeurs aux bornes du mot dans la phrase mélodique (point de jonction entre l'intonation ou mélodie de la phrase, et le mot), indiquaient soit :

- un plateau, fichiers son $\mathrm{n}^{\circ}$ 3-4, 9-10 (ex : niveau /bb =33/, /grand-mère/, /moi/),

- une pente montante, fichiers son $\mathrm{n}^{\circ} 1-2,7-8,9-10(\mathrm{ex}: / \mathrm{ic}=24 /, /$ partante $/ \mathrm{bc}=$ 34/, premier /très/, /poissons/),

- une pente descendante, fichiers son n 5-6, 7-8, 9-10 (ex: /bg = 31/, /Soulac/; $/ \mathrm{cb}=$ 43/, deuxième /très/, /souvent/).

Dans l'exemple d'un même énoncé ( $\mathrm{n}^{\circ} 9$ et 10$)$, nous avons modifié la courbe sonore en insérant 3 mélismes : /moi, souvent, poissons/.

\section{Position des mélismes par rapport au groupe}

Nous avons tenté d'illustrer les mélismes dans les diverses configurations par rapport au groupe :

-MP, en fin de groupe devant pause : /Soulac/, /poissons/,

- MF, en fin de groupe sans pause : /grand-mère/, /moi/),

- M, en position interne de groupe :/partante/, très/, /très/, /souvent/.

Les mélismes par contact MC, jugés moins intéressants, n'ont pas été retenus.

\section{Méthode}

Nous avons réalisé cette resynthèse à l'aide du logiciel Praat (Boersma and Weenink, 2012). Les principales étapes sont résumées ci-dessous.

4.1. Sélection des mots (cf ci-dessus) et des phrases dans la base de données des sons

4.2. Extraction de la phrase souhaitée sous Praat, et obtention des fichiers texte (segmentation et étiquetage des mots) appelés « TextGrids »

\subsection{Obtention du fichier " Manipulations »}

Il est obtenu de manière automatique grâce à un script, intégré dans PRAAT-MOMEL (Hirst, 2007), script que j'ai conçu et qui a été entièrement réécrit et augmenté de nouvelles fonctionnalités en 2012 par un ingénieur de l'Institut MICA (MISTRAL +, Weber et al., 2012). 


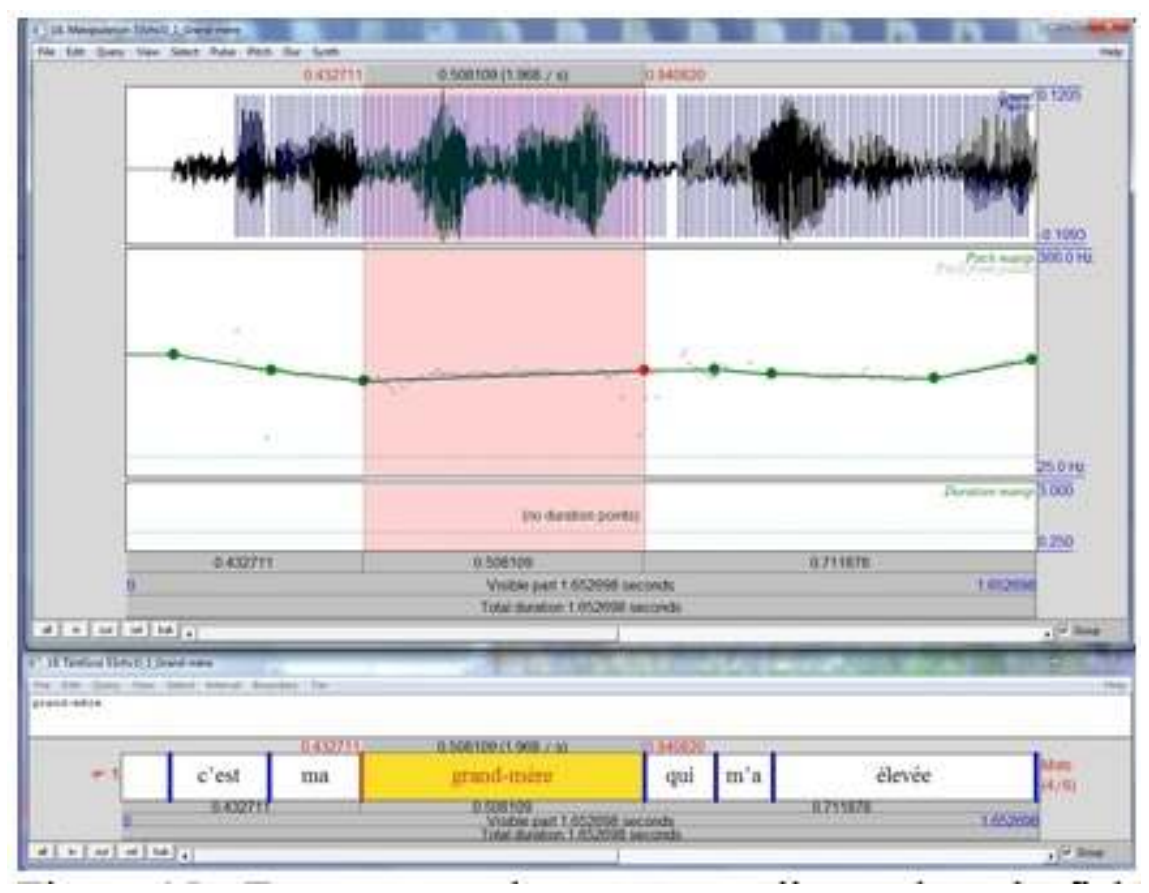

Figure 15 : En correspondance temporelle, en haut le fichier « Manipulations », en bas le « TextGrid » de segmentation et annotation, signal original, étape 1 : ajout, suppression, ou déplacement des points (en vert ou rouge) pour alignement (ici opéré de manière manuelle) avec les barres de segmentation du TextGrid. On remarque que la ligne en vert et les points se trouvent sur le tracé de la courbe mélodique (petits points gris) Lien vers exemple sonore 3 original.

Ce fichier Manipulations a la propriété de créer une courbe mélodique à partir du signal de parole traité par Praat, que l'on peut modifier à la main, grâce à l'ajout, suppression, déplacement de « points » correspondants à une hauteur mélodique, comme le montre la Figure 15 ci-dessus.

Le script MISTRAL+ est ensuite activé, de manière à nous fournir toutes les informations prosodiques (mélodiques, temporelles, dérivée de la mélodie...) que l'on désire.

Au niveau mélodique, tous les registres des locuteurs -soigneusement évalués lors de l'analyse initiale de recherche et traitement des 400 mélismes issus de la banque de données des sons- ont été comme on le sait divisés en 9 niveaux, exprimés en Hz et en demi-tons. C'est sur la base d'une échelle en demi-tons que nous pouvons établir leur conversion en 9 niveaux (échelle lettres ou numérique), comparer les mélismes entre locuteurs de registres différents, et leurs réalisations.

\subsection{Modification des patrons mélodiques pour créer les mélismes}

A l'aide des valeurs mélodiques (appelées cibles mélodiques) correspondant en particulier aux barres de segmentation, nous pouvons déterminer si le mot original (non mélismé) possède une pente ascendante, descendante ou si c'est un plateau, et la hauteur de ces cibles. Par exemple dans le cas de notre exemple /grand-mère/, il s'agit d'un plateau avec les cibles /bb/. Pour respecter le naturel, le mélisme à créer s'inscrira dans le type de pente originel : rien n'est modifié à ce niveau. 


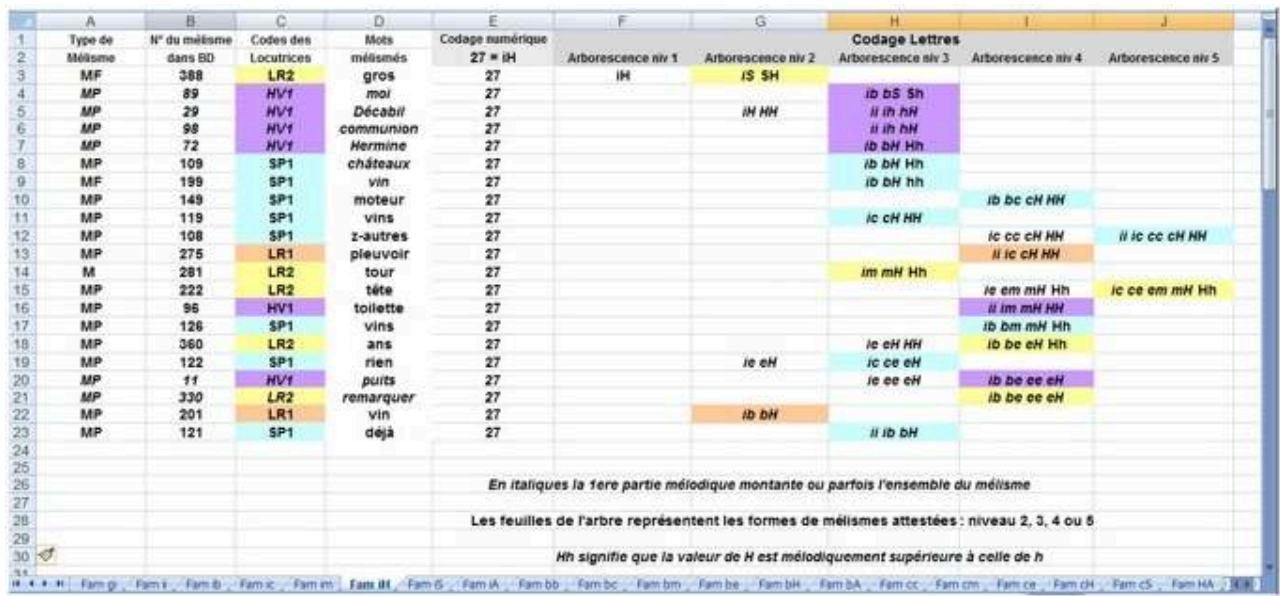

Tableau 11 : Extrait du dictionnaire de formes mélodiques montantes de mélismes : ici l'exemple de la famille « $\mathrm{iH}=27$ ", avec respectivement de la colonne A à la colonne $\mathrm{E}$, toutes ses occurrences identifiées par le type de mélisme, son numéro dans la base de données, la référence de la locutrice, les mots mélismés, le code numérique de la famille, puis de la colonne $\mathrm{F}$ à la colonne J, l'arborescence développant 5 niveaux. Les feuilles de cet arbre représentent les formes de mélismes attestées. Les couleurs renvoient aux locutrices. Tout en bas, la suite des feuilles excel offre par familles, toutes les formes des mélismes ascendantes attestées dans le corpus.

Munie de cette information, nous avons consulté le dictionnaire des mélismes (cf Tableau 11 ci-dessus). Ce dernier comporte deux parties correspondant aux mélismes mélodiquement croissants et décroissants. Elles sont organisées de la même façon : les mélismes sont distribués en fonction des valeurs mélodiques de début et fin de mot (appelées familles, codées sous forme de lettres représentant les 9 niveaux qui partagent le registre mélodique de toutes les locutrices, soit $/ A=9, S=8, H=7, e=6, m=5, c=4, b=3$, $\mathrm{i}=2, \mathrm{~g}=1 /$ ).

Dans celui-ci, les mélismes sont décrits sous forme d'arborescence. Les mélismes attestés correspondent aux feuilles des arbres (cellule en couleur en fonction du locuteur), qui peut éventuellement correspondre aussi à la racine (ex : /SS/).

La présentation en arbres a plusieurs avantages. Elle permet en effet de :

- répertorier les mélismes proches soit par filiation, soit par frérie,

- choisir des patrons mélodiques plus ou moins complexes, appartenant à la même famille et sous-famille, par exemple en fonction de la durée du mot,

- créer des patrons mélodiques non attestés en fonction des «trous" dans l'arborescence.

Dans le cas du mot /grand-mère/, sa famille est /bb/. Dans la feuille excel correspondante, nous avons trouvé 2 mélismes et avons choisi le mélisme possédant des contrastes mélodiques internes les plus forts /bbHeib/, dus à des oppositions de direction de pente $/ e \neq i \neq b /$, bien visibles Figure 2 ci-dessous, par opposition à / $\mathrm{bcHecb} /$ qui montre de simples changements d'angles de pentes.

Le choix peut aussi être affiné en fonction de la nature lexicale ou non du mot à mélismer, de sa position par rapport au contexte, cf Tableau 11, colonne A :

- MP : mélisme en fin de groupe ou phrase devant pause

- MF : en fin de groupe sans pause subséquente, 
- M : mélisme en position interne,

- MC : mélisme par contact. Il s'agit d'un mot grammatical, (le plus souvent en position antérieure), qui est réalisé en mélodie haute du fait du véritable mélisme qui le suit.

Ce dictionnaire peut bien entendu être chaîné avec la base de données accédant ainsi à toutes les informations prosodiques qui concernent les mélismes candidats, en particulier la durée qui peut contribuer avec l'énergie à augmenter la perception de la proéminence.

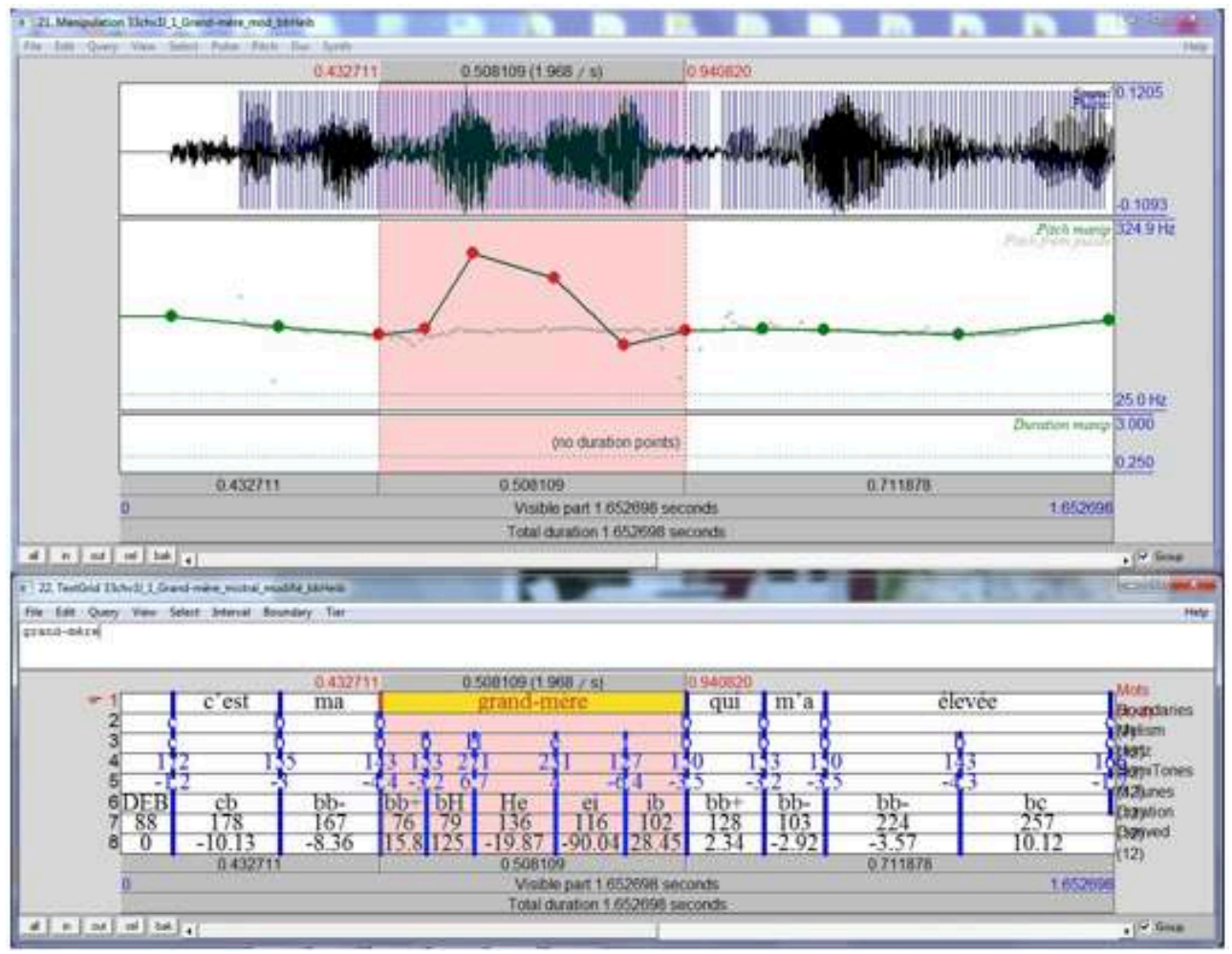

Figure 16 : Fichier Manipulations et TextGrid. Chaque point du fichier Manipulations a donné lieu à un calcul automatique des valeurs correspondantes (ligne 2 et 3 respectivement, valeurs des cibles en échelle lettres de 9 niveaux aux bornes des mots, et aux points de modification de la courbe mélodique ; ligne 4 , valeurs correspondante en $\mathrm{Hz}$; ligne 5, en demi-tons. Ligne 6, l'espace dans le mélisme entre 2 cibles (c'est-àdire entre 2 points) est appelé « syllabe mélodique ", et correspond à une information en dynamique de la pente (montée, descente, plateau). Les signes «+ » ou « - » indiquent relativement à un plateau de cibles $(\mathrm{ex}: / \mathrm{bb}+, \mathrm{bb}-/)$ une pente lorsqu'elle est perceptible ( $3 \%$ de modification de la pente). La ligne 7 indique la durée de ces syllabes mélodiques et la ligne 8 , leur valeur de dérivée concernant la mélodie (Lien vers exemple sonore 4 mélismé).

A l'aide de ces valeurs mélodiques offrant un patron de mélisme approprié, et leurs correspondances exprimées en $\mathrm{Hz}$ et demi-tons, nous avons placé manuellement les points dans le fichier Manipulations. Lançant à nouveau le script MISTRAL+, nous avons obtenu le nouveau TextGrid avec toutes les valeurs calculées de manière automatiques, comme le montre la Figure 16 ci-dessus.

Par comparaison avec la Figure 15, ou Figure 16 par le simple tracé original de la mélodie en petits points gris, et bien entendu par l'écoute des fichiers (Lien vers 
exemples sonores original et modifié 3 et 4), nous pouvons juger de la différence des patrons mélodiques, très sensible visuellement comme auditivement.

\subsection{Resynthèse}

La resynthèse des exemples proposés est obtenue grâce à Praat qui propose depuis le fichier Manipulations la création d'un fichier son. Nous précisons que seule la mélodie a été modifiée comme indiqué ci-dessus, et qu'en particulier nous avons conservé les durées.

Ce que nous proposons à l'évaluation est non pas la resynthèse qui est celle de Praat (bien que d'un bon niveau, dans certains exemples il y a malgré tout parfois quelques menues discordances acoustiques), mais la hauteur mélodique modifiée qui a fait d'un mot sans expressivité ni mise en relief mélodique, un mot mélodiquement proéminent, comme nous l'avons constaté au paragraphe 6 ci-dessus dans la communication (Lien vers exemples sonores original et modifié 1 et 2). Comme nous pouvons l'entendre plusieurs mots peuvent être également mélismés dans un même énoncé soit à la suite l'un de l'autre (Lien vers exemples sonores original et modifié 7 et 8 , /très très/), soit même à 2 ou 3 mots près (Lien vers exemples sonore original et modifié 9 et 10, /moi/ / souvent/ / poissons/).

Tous ces exemples sonores montrent que les énoncés acquièrent plus d'expressivité, ils sont en quelque sorte appropriés par le locuteur, renforçant parfois une attitude comme dans l'exemple / Soulac/, (Lien vers exemples sonores original et modifié 5 et 6), ici celle d'évidence et de résignation. On remarquera là aussi la diversité des patrons, montrant ainsi que l'on peut créer une synthèse vivante et non monotone.

Bien entendu toutes les opérations décrites ont été faites de manière manuelle, mais elles sont toutes automatisables par les ingénieurs qui travaillent en génération et synthèse de la parole.

\section{Conclusion}

Comme cette expérience le montre, cette technique est applicable soit à de la parole naturelle que l'on souhaite modifier (en mettant en relief certains mots ou au contraire en les rendant mélodiquement plus neutres), soit à de la parole synthétique.

\section{NOTES}

1. Toutefois il est important d'apporter une précision : cette étude se présente comme une étude prosodique située en amont de la génération de parole. Il ne saurait donc s'agir de lier cette étude aux systèmes de synthèse existants, mais au contraire à évaluer ses applications à la synthèse en collaboration avec des spécialistes du domaine.

2. Pour les analyses linguistiques et pragmatiques des mélismes, le lecteur s'orientera vers les références citées ci-dessus et en particulier 2009a, un ouvrage qui reprend d'une part les principaux résultats de ma thèse d'état (comparaison de 6 modèles linguistiques, syntaxiques, sémantiques, pragmatiques, prédictifs des hauteurs mélodiques, et mis méthodologiquement en concurrence pour expliquer les modulations mélodiques dans les unités lexicales et grammaticales), et d'autre part fait état du prolongements de ces recherches jusqu'en 2007. Plusieurs types de corpus ont été ainsi utilisés en vue de ces analyses. 
3. Un diphone est un petit segment de parole commençant généralement au milieu d'un phonème jusqu'au milieu du phonème suivant, ce qui permet de régler le délicat problème des transitions voyelle / consonne / voyelle en synthèse.

4. Une « tire » est une ligne d'étiquetage manuel ou automatique dans un TextGrid de Praat.

5. La nouvelle procédure MISTRAL+ ayant entièrement réécrit les versions antérieures (INTSMEL puis MELISM), permet désormais de choisir un codage alphabétique ou numérique, et le nombre de niveaux (de 2 à 9) partageant le registre mélodique du locuteur. Cette nouvelle procédure MISTRAL+ s'adapte ainsi à tous les types d'études, à toutes les langues, à accent libre, accentuelles ou tonales, et aux besoins des utilisateurs.

6. Les différentes appellations des parties au sein du mélisme sont symétriques (ex : pied pour les cibles externes du mélisme -ou du Noyau-, corps pour les cibles plus internes).

7. Cette structure " phonologique » des 7 cibles types, peut en contenir en fait davantage dans la mesure où les cibles des corps (notamment / Nc/ et / Ac/) peuvent être dupliquées, cf le Tableau 2 et les commentaires.

8. Dans le cas de valeurs identiques, nous avons classé en 2 cibles possibles (cf par exemple Ac ou $\mathrm{Nc}$ ), et ensuite réparti les effectifs correspondants au prorata de Ac et Nc par rapport à la somme totale de leurs effectifs $(\mathrm{Ac}+\mathrm{Nc})$.

9. Dans le domaine automatique, on peut citer pour information et sans développer davantage, les techniques d'annotation sémantique qui peuvent détecter des éléments atomiques signifiants, tels que les noms de personnes, d'organisations, les acronymes, lieux, dates, etc., et maintenant les mots-clefs de domaines. Par ailleurs les chaînes lexicales empruntées au domaine du résumé automatique, ou bien les travaux sur la structure et intentionnelle (ou rhétorique) qui coexistent dans le discours, permettent d'envisager la sélection du vocabulaire pour les usagers de la synthèse en fonction de buts (informationnels, didactiques, ou un mixte des deux). D'autres notions comme celles de mesure de la saillance des unités en fonction de leur position, de nucléarité (ou évaluation de l'importance relative -noyau vs. satellite- que l'auteur de l'énoncé accorde à deux éléments reliés), de pertinence permettant de calculer et de sélectionner les termes fréquents et spécifiques à un document, laissent entrevoir à court ou moyen terme, des applications innovantes à la génération et synthèse de la parole telles qu'on les envisage dans ces pages, rejoignant ainsi de plus en plus la finesse et la précision des études linguistiques.

10. Ces pourcentages sont présentés sous forme de courbe pour mieux voir l'évolution des effectifs d'une locutrice à une autre.

11. Comme déjà précisé dans la texte de la communication présente, les petites unités appelées «diphones» ne sont pas seulement des voyelles et des consonnes. En effet le problème du traitement acoustique des transitions entre voyelle / consonne / voyelle s'étant révélé extrêmement ardu, les chercheurs se sont acheminés vers des larges bases de données contenant des unités phonétiques mixtes commençant au centre d'une voyelle pour se terminer dans la suivante, englobant donc le ou les consonnes, et résolvant ainsi le problème des transitions.

\section{RÉSUMÉS}

Le travail présenté ici est issu d'une expertise linguistique. Il se propose d'aborder une nouvelle façon de traiter l'expression subjective en vue d'une exploitation en synthèse vocale. Le traitement linguistique de la prosodie subjective par les mélismes constitue l'essentiel de ce travail qui aborde de manière exhaustive tous les éléments essentiels de ces structures 
mélodiques, vues de manière externe par rapport à leur localisation, leurs critères généraux, et de manière interne sous plusieurs angles. Dans la perspective prosodique, nous aborderons à la fois les points de vue mélodique et temporel afin d'offrir les critères fondamentaux permettant de manière conforme à la voix naturelle de reproduire des mélismes dans la voix de synthèse.

The work presented here issued from a linguistic expertise, is intended to address a new way of processing the subjective expression in the perspective of the voice synthesis. The linguistic processing of the subjective prosody constitutes much of this work, trying to deal comprehensively with all elements of these melodic structures, seen in an external way with regard to their localization, their general criteria, and in an internal way, to several angles. In the prosody domain, we will approach at the same time the melody and temporal points of view in order to conform to the natural voice and make it possible to reproduce melisms as such in the vocal synthesis.

INDEX

Keywords : subjectivity, voice synthesis, melism, melodic syllables, F0, duration, patterns, living melody

Mots-clés : subjectivité, synthèse, vocale, mélisme, syllabes mélodiques, F0, durée, patrons, mélodie naturelle

\section{AUTEUR}

\section{GENEVIÈVE CAELEN-HAUMONT}

MICA Institute, UMI CNRS 2954, Hanoi University of Technology 